\title{
An Automated Method for Depicting Mesocyclone Paths and Intensities
}

\author{
Madison L. Miller ${ }^{1}$ \\ Cooperative Institute of Mesoscale Meteorological Studies, University of \\ Oklahoma and National Severe Storms Laboratory, Norman, Oklahoma \\ Valliappa Lakshmanan
}

Cooperative Institute of Mesoscale Meteorological Studies, University of Oklahoma and National Severe Storms Laboratory, Norman, Oklahoma

Travis M. Smith

Cooperative Institute of Mesoscale Meteorological Studies, University of Oklahoma and National Severe Storms Laboratory, Norman, Oklahoma

(1)


Abstract

42 The location and intensity of mesocyclone circulations can be tracked in real-time by

43 accumulating azimuthal shear values over time at every location of a uniform spatial grid.

44 Azimuthal shear at low (0-3 km AGL) and mid levels (3-6 km AGL) of the atmosphere is

45 computed in a noise-tolerant manner by fitting the Doppler velocity observations in the

46 neighborhood of a pulse volume to a plane and finding the slope of that plane. Rotation

47 tracks created in this manner are contaminated by non-meteorological signatures caused

48 by poor velocity dealiasing, ground clutter, radar test patterns and spurious shear values.

49 In order to improve the quality of these fields for real-time use and for an accumulated

50 multi-year climatology, new dealiasing strategies, data thresholding, and Multiple

51 Hypothesis Tracking (MHT) techniques have been implemented. These techniques

52 remove nearly all non-meteorological contaminants resulting in much clearer rotation

53 tracks that appear to match mesocyclone paths and intensities closely. 


\section{$54 \quad 1 . \quad$ Introduction}

\section{a. Motivation}

56 To depict the paths and intensities of mesocyclone circulations as seen by radar,

57 the National Severe Storms Laboratory (NSSL) creates products called "rotation tracks".

58 These rotation track fields are created by calculating the azimuthal shear fields (the

59 azimuthal derivative of radial velocity) for each radar, merging the azimuthal shear data

60 from multiple radars onto Cartesian grids and then accumulating the maximum values in

61 those gridded fields over time onto one accumulated grid. An example is shown in Figure

621.

63 These tracks can help alleviate some of the difficulty in interpreting and analyzing

64 velocity fields. They can provide information about the spatial extent and strength of

65 mesocyclone signatures over time and can be quite useful for conducting poststorm

66 damage surveys. The American Red Cross of Central Oklahoma uses NSSL rotation

67 track products plotted on maps to determine where to deliver assistance after tornado

68 events and the best routes to get there. After the 24 May 2011 tornado outbreak in Central

69 Oklahoma, the use of rotation tracks helped to significantly reduce the disaster

70 assessment time (NOAA/NSSL 2011). These fields are also useful in real-time as

71 guidance for forecasters and have enormous data mining potential. However, they are

72 plagued by non-meteorological signatures caused by poor velocity dealiasing, ground

73 clutter, radar test patterns, and spurious shear values. As seen in Figure 2, these artifacts

74 can make the tracks difficult, if not impossible, to interpret meaningfully.

75 One of the goals of the Multi-Year Reanalysis of Remotely Sensed Storms

76 (MYRORSS) project, a cooperative effort between National Oceanic and Atmospheric 
77 Administration's (NOAA) NSSL and the National Climatic Data Center (NCDC), is to

78 create a CONUS-wide climatology of low and mid level rotation track fields for the

79 lifetime of the Weather Surveillance Radar 1988-Doppler (WSR-88D) network.

80 Cintineo et al. (2011) developed an automated system to process Level-II radar

81 data (Crum and Alberty 1993) at NSSL using a multiple-machine framework and the

82 Warning Decision Support System - Integrated Information (WDSS-II; Lakshmanan et

83 al. 2007b) suite of programs to process and quality control the data. A preliminary hail

84 climatology using Maximum Estimated Size of Hail (MESH) grids was also created.

85 Here, we extend the processing to velocity-based products, specifically to azimuthal

86 shear accumulations.

87 The CONUS-wide rotation track climatology will provide an incredibly rich

88 dataset with numerous potential climatological and severe weather applications. Track

89 lengths, intensities, and other characteristics could be analyzed by geographical region

90 and time of year. Potential relationships could also be discovered between rotation tracks

91 and environmental parameters like convective available potential energy (CAPE) and

92 storm-relative helicity (SRH). After correlating maximum azimuthal shear and maximum

93 updraft helicity, rotation tracks could also be used as verification for high-resolution

94 model-simulated maximum updraft helicity tracks like the ones discussed by Kain et al.

95 (2010) and Clark et al. (2012).

96 The purpose of this paper is to discuss the special velocity dealiasing techniques,

97 data thresholds, and Multiple Hypothesis Tracking (MHT) techniques developed to

98 isolate the rotation tracks in real-time and for the MYRORSS climatology. Detailed 
99 explanations of each quality control effort will be given and the specific impacts of each

100 step will be shown in example cases.

102 Modern Doppler radars have the ability to provide high resolution space and time

103 measurements of storms that allow for the detection of mesocyclone-scale circulations.

104 Couplets in radial velocity fields as well as hook echo signatures in reflectivity fields

105 have been used to identify these circulations in the past, but methods of identifying

106 mesocyclone or tornado circulations reliant solely on hook echo signatures have proven

107 unreliable (Forbes 1981; Mitchell et al. 1998). Methods using radial velocity signatures

108 such as the Tornado Detection Algorithm (TDA; Mitchell et. al 1998) and the NSSL

109 Mesocyclone Detection Algorithm (MDA; Stumpf et. al 1998) have been more

110 successful.

111 The TDA currently used with the WSR-88D system relies on high "gate-to-gate

112 velocity difference" values to identify potentially tornadic circulations (Mitchell et al.

113 1998). Although termed a tornado detector, the algorithm identifies tornadic vortex

114 signatures (which may or may not be associated with tornadoes) that are typically larger

115 than a tornado owing to radar sampling resolution (e.g., Brown et al. 1978). The gate-to-

116 gate difference represents the difference between velocity values at constant range from

117 the radar between adjacent azimuths. These values can be affected adversely by the

118 azimuthal offset of the radar beam center from the vortex, noisy data, and velocity

119 aliasing (Wood and Brown 1997). Additionally, because the radar beam is much broader

120 at far ranges than when near the radar, observed velocity peaks within vortices decrease

121 in magnitude, allowing some vortices to be overlooked by the algorithm. 
123 issues. The method examines region-to-region radial wind shears at a number of different

124 spatial scales to more accurately determine the amount of shear present, reducing the

125 number of false tornado detections.

126 Rather than using the gate-to-gate velocity difference, "peak-to-peak" methods of

127 calculating rotational shear from Doppler radial velocity data or the wavelet analysis

128 technique to detect a vortex, a two-dimensional, local, linear least squares derivatives

129 (LLSD) method can be used to reduce the impact of noise. Elmore et al. (1994) proposed

130 this method of estimating the derivatives of radial velocity values by fitting a plane to the

131 velocity field and finding its slope. The vertical vorticity field is estimated by the

132 azimuthal derivative of the radial velocity field and is given by

$$
\frac{\partial V_{r}}{\partial s}=\frac{\sum s_{i j} V_{i j} \omega_{i j}}{\sum\left(\Delta s_{i j}\right)^{2} \omega_{i j}}
$$

133 where $V_{r}$ is the radial velocity, $s$ is the coordinate in the azimuthal direction, $s_{i j}$ is the arc

134 length from the center point of the calculation to the point $(i, j), V_{i j}$ is the radial velocity

135 at point $(i, j)$, and $\Delta s_{i j}$ is the beam width at a given range. $\omega_{i j}$ is a positive weight

136 function that we set to 1 after determining that Cressman weight functions, among others,

137 generated very little differences. The coordinate $i$ is in the radial direction and $j$ is in the

138 azimuthal direction. The summation is performed over range gates in the neighborhood

139 of the starting point of the calculation. This calculation of azimuthal shear is, here on,

140 referred to as the LLSD method.

141 Smith and Elmore (2004) applied the LLSD calculation to simulated and observed

142 circulations by first passing the velocity data through a $3 \times 3$ median filter (Lakshmanan 
143 2012) to reduce speckle noise and then applying Eq.1 to the filtered velocity data to

144 estimate the azimuthal shear. The physical size of the neighborhood used in the

145 calculation is held constant such that fewer radials are used in the calculation at far

146 ranges from the radar. Typical sizes of radial and azimuthal neighborhoods are $750 \mathrm{~m}$ and $1472500 \mathrm{~m}$, respectively.

148 The LLSD calculation helps to remove some of the dependence on radar location

149 involved in rotation detection and also allows circulation signatures to be viewed in

150 three-dimensional space or as input to multi-sensor applications. It was shown by Smith

151 and Elmore (2004) that LLSD shear values were reasonable estimations of actual shear

152 values in simulated Rankine vortices when sampled by a theoretical WSR-88D radar

153 (Brown et al. 2002) out to a range of $\sim 140 \mathrm{~km}$. The variance of these values was also

154 much smaller compared to peak-to-peak shear.

155 The use of the median filter in the LLSD shear technique can be a disadvantage,

156 however. Whereas areas with large velocity gradients are preserved, this filter can also

157 smooth out peaks in the velocity field. Although the median filter is beneficial when

158 these peaks are associated with noisy data, the filter decreases the magnitude of peak

159 velocities in mesocyclone signatures and completely eliminates tornado signatures in

160 nearly all cases. For this reason, LLSD shear values may underestimate the actual

161 azimuthal shear of circulations, especially for small circulations (Mitchell and Elmore 162 1998).

163 Defining a neighborhood size in the LLSD technique also forces a trade-off

164 between spatial resolution and noise resistance. Smaller neighborhoods are more strongly

165 affected by noise, whereas larger neighborhoods tend to underestimate actual shear 
166 values of circulations. The spatial scale of the LLSD calculation makes it most useful for

167 detecting large mesocyclone-scale circulations, however, the increased noise resistance

168 makes identification of small circulations more difficult.

169 Rotation tracks help to visualize the movement of mesocyclone circulations (or

170 occasionally circulations associated with nearby large tornadoes) over time as seen by

171 radar. To produce these tracks, radial velocity data are first dealiased using the default

172 WSR-88D dealiasing algorithm (Eilts and Smith 1990). Next, a quality control neural

173 network (Lakshmanan et al. 2007a) is used to remove echoes in the reflectivity field

174 produced by biological targets, anomalous propagation, ground clutter, and test or

175 interference patterns. The algorithm successfully removes nearly all non-meteorological

176 signatures from the Reflectivity fields examined.

177 This quality controlled reflectivity field, hereafter ReflectivityQC, and the radial

178 velocity field are then employed by the shear estimation algorithm of Smith and Elmore

179 (2004) to compute the azimuthal shear (see Fig. 3). Two-dimensional maximum

180 azimuthal shear fields within low $(0-3 \mathrm{~km})$ and mid $(3-6 \mathrm{~km})$ level layers above ground

181 level (AGL) are also calculated using digital elevation model (DEM) data to determine

182 the height of each point above the ground.

183 These single-radar 2-D maximum azimuthal shear fields are then merged into a

184 Cartesian multi-radar grid using the intelligent agent formulation of Lakshmanan et al.

185 (2006), accounting for varying radar beam geometry with range, vertical gaps between

186 radar scans, and other issues. The maximum value of each pixel in the merged multi-

187 radar grid over a time interval (typically 60 -120 minutes) is then used to produce the

188 swaths of merged maximum azimuthal shear known as rotation tracks. Missing data in 
189 the rotation track fields (denoted by "MD" in the color bar) corresponds to any data

190 below the signal to noise threshold for the radar. Ideally, this should be shown as zero

191 shear, not missing data. Figure 4 provides a flow chart showing the algorithms and fields

192 used to create rotation tracks.

193 2. Methods

\section{a. Two-dimensional velocity dealiasing}

195 Due to the relationship between radar wave length $(\lambda)$ and the pulse repetition

196 frequency $(F)$, a radar correctly measures the radial velocity given that it is in the range

197 of $\pm V_{N}$, where

$$
V_{N}=\frac{\lambda F}{4}
$$

198 Here, $V_{N}$ is the Nyquist velocity and the true velocity is

$$
V=V_{m} \pm 2 n V_{N}
$$

199 where $V_{m}$ is the measured velocity, $n$ is an unknown integer including zero, and $V_{m}$ must

200 satisfy $-V_{N} \leq V_{m} \leq V_{N}$. Velocity dealiasing is the process of determining the correct

201 value of $\mathrm{n}$ to successfully recover $V$. In the cases when $V$ cannot be successfully

202 recovered, the velocity is still aliased and can usually be identified in radial velocity

203 fields by abrupt changes in values between neighboring measurements. Most first

204 generation dealiasing algorithms (e.g. Ray and Ziegler 1977, Bargen and Brown 1980)

205 were one-dimensional and detected abrupt changes between single radials. For this

206 reason, they were quite sensitive to noisy, incorrect data. Strong shear zones in these

207 radial velocity fields sometimes cannot be dealiased without data from multiple 
208 dimensions. Merritt (1984), Boren et al. (1986), and Bergen and Albers (1988) took more

209 sophisticated approaches and used velocity data in two dimensions to dealias. These

210 methods were costly in terms of computation time, however.

211 The local environment dealiasing (LED) algorithm (Eilts and Smith 1990) is the

212 method currently used for WSR-88D data in real-time. The scheme applies radial

213 continuity constraints to remove local aliasing errors and azimuthal continuity checks to

214 mitigate error. It also incorporates radial averages to determine $n$ (see Eq. 3) when

215 continuity thresholds are not met. Each radial is processed individually and compared

216 against the previously dealiased radials, allowing the algorithm to use less memory and

217 process faster than other two-dimensional algorithms. It can also ingest a vertical wind

218 profile from an environmental sounding to produce initial values for each elevation scan

219 and for isolated echoes. It is an efficient algorithm that begins by using simple checks and

220 only moves on to more sophisticated techniques if needed. This approach performed very

221 well on the cases of severe aliasing presented in Eilts and Smith (1990), but performed

222 poorly when qualitatively examined in many of the tornadic cases examined for this

223 study. Ingesting the vertical wind field from a 20-km Rapid Update Cycle model (RUC;

224 Benjamin et al. 2004) point sounding at each radar site to use as an environmental

225 estimate into the LED algorithm improved the dealiasing to some extent.

226 For this study, a sophisticated two-dimensional dealiasing technique described by

227 Jing and Wiener (1993) was implemented. The algorithm solves a linear system of

228 equations that minimizes gate-to-gate shear in each isolated two-dimensional region.

229 Through using aliasing-induced discontinuity information, the correction values for all

230 gates are found by solving a two-dimensional least-mean-squares problem. Instead of 
231 making dealiasing decisions for each gate based on its neighbors, which can be subject to

232 scattered incorrect data, this approach avoids local expansion of errors by attempting to

233 find all dealiased values for a given dataset. Vertical profiles of horizontal wind data

234 from the 20-km RUC point soundings were used as environmental wind estimates at the

235 grid-point nearest to each radar site. The calculated average is minimized by

236 incrementing $n$ equally over the entire echo. The average local wind observed by radar

237 is assumed to be less than $V_{n}$.

238 A smooth environmental wind field with weak shear is assumed. This can be a

239 poor assumption in isolated areas of strong wind shear associated with mesocyclones or

240 microbursts. In these cases, relatively short falsely aliased border segments are detected

241 and can typically be used to dealiase the field correctly. In more elongated regions of

242 shear associated with strong gust fronts, for example, incorrect dealiasing is more likely.

243 Example dealiased radial velocity fields and their corresponding azimuthal shear fields

244 using both the LED and Jing and Weiner (1993) methods are shown in Fig. 3.

245 Preliminary results from a study now underway to determine which velocity dealiasing

246 method performs best on a set of case studies indicate that this two-dimensional

247 technique is more accurate than the LED technique.

$248 \quad$ b. Reflectivity quality control

249 As mentioned in the introduction, the quality control neural network

250 (Lakshmanan et al. 2007b, Lakshmanan et al. 2010) is used to remove non-

251 meteorological echoes from the reflectivity field. The algorithm combines various

252 measures from both past literature (e.g. Steiner and Smith 2002, Kessinger et al. 2003,

253 Fulton et al. 1998) and new measures to discriminate between precipitating and 
254 nonprecipitating echoes in the reflectivity data. A region-by-region classification is

255 performed rather than a gate-by-gate basis. In addition, clear air echoes due to biological

256 contamination are identified and removed using a two-stage intelligent machine

257 algorithm while retaining echoes that correspond to precipitation (Lakshmanan et al.

258 2010).

c. Additions to LLSD shear algorithm

260 In addition to calculating the azimuthal shear fields as described earlier in this

261 paper, extra operations have been added to the LLSD algorithm. These additions are

262 discussed in the following sections.

\section{1) AZIMUTHAL SHEAR RANGE CORRECTION}

A new azimuthal shear range-correction (Newman et al. 2012) algorithm is

265 applied to the field in an effort to correct for range degradation due to radar beam

266 widening. A multiple linear regression technique was used to create the equations based

267 on comparisons between observed shear values and those computed using simulated

268 Rankine vortices. First, the algorithm identifies significant circulations using reflectivity

269 and LLSD shear criteria. To avoid applying the range-correction to regions of noise in the

270 shear fields co-located with low reflectivity values, only circulations in regions with

271 reflectivity values greater than $20 \mathrm{dBZ}$ and LLSD shear values exceeding $0.005 \mathrm{~s}^{-1}$ are

272 identified. The peak-to-peak velocity differences and shear diameters of circulations

273 satisfying the reflectivity and shear criteria are calculated next. A median filter is applied

274 to the shear diameter field to provide potentially more accurate estimates of circulation

275 size when circulations are larger than tornadic vortex signatures (TVSs; Brown, Lemon, 
276 and Burgess 1978). Then, new azimuthal shear values for each pixel in the circulations

277 are calculated by inserting the associated shear diameter, maximum velocity measured,

278 and range values into the appropriate regression equations. Newman et al. (2012) found

279 that the algorithm increased tornadic shear values appropriately and aided in the

280 differentiation between tornadic and nontornadic scans.

2) DATA THRESHOLDS AND REMOVAL

Significant vertical shear near the surface can cause false high azimuthal shear

283 values very close to the radar. To prevent these high values from corrupting the multi-

284 year climatology, azimuthal shear values within a 5-km radius of each radar site are set to

285 'missing'. While this will remove some 'good' data, it will also remove a great deal of

286 anomalously high azimuthal shear values that could corrupt the climatology. An example

287 is shown in Figure 5. This near-radar data removal is not applied to the rotation tracks in

288 this paper, nor will it be used in generation of rotation tracks in real-time. It is used only

289 in the climatology to avoid accumulations at areas where the azimuthal shear values are

290 known to be poor.

291 When processing the two-dimensional maximum azimuthal shear fields, all shear

292 data not co-located with a given ReflectivityQC value are removed so that only shear data

293 associated with storm regions are retained. In order to retain meteorologically significant

294 shear data in low reflectivity hook echo regions, a 5x5 dilation filter (Lakshmanan 2012)

295 is applied to the ReflectivityQC field. This operation assigns the maximum reflectivity

296 value in a 5x5 moving window to each pixel, effectively expanding the areas of high

297 reflectivity values. The threshold operation is then performed on the dilated

298 ReflectivityQC field to help remove azimuthal shear associated with interference 
299 patterns, anomalous propagation, and other radar-related issues not successfully removed

300 by the radar reflectivity quality control neural network. Setting this threshold at $40 \mathrm{dBZ}$

301 retained the meteorological rotation signatures in the azimuthal shear fields while

302 removing a great deal of shear co-located with non-meteorological signatures (see Figure

303 6).

d. Creation of rotation tracks

305 To better isolate the rotation tracks in the accumulated grid, new quality control

306 strategies have been implemented on the input two-dimensional maximum azimuthal

307 shear fields for both low and mid levels of the atmosphere. Clusters of high azimuthal

308 shear values in each time step are tested and removed if their sizes and/or data value

309 distributions are not indicative of meteorological phenomena. If these remaining clusters

310 in each time step are associated with lasting circulations, they make it into the final

311 rotation track products.

313 Before the circulation signatures in the two-dimensional maximum azimuthal

314 shear fields can be associated between time steps, they are isolated into clusters of high

315 shear values using hysteresis segmentation. The term hysteresis (Jain 1989) refers to the

316 lag observed between the application of an electromagnetic field and its subsequent effect

317 on a substance. In image processing, the term refers to the lagging effect caused by using

318 two thresholds - one to begin the thresholding process and another to end it. In this

319 application, two data thresholds are maintained and a cluster is composed of contiguous

320 pixels with values greater than the lower data threshold that contains at least one pixel 
321 with a data value greater than the higher threshold. The higher threshold identifies areas

322 of high azimuthal shear associated with strong circulation and the lower hysteresis

323 threshold grows the region around the high value to include all pixels associated with the

324 circulation (see Figure 7). Through experimentation on numerous tornadic and

325 nontornadic case studies, it was determined that low and high data thresholds of $0.002 \mathrm{~s}^{-1}$

326 and $0.005 \mathrm{~s}^{-1}$, respectively, and a minimum size of 25 pixels performed well for isolating

327 clusters of high azimuthal shear.

328 All pixels in the maximum 2-D azimuthal shear layer fields not associated with

329 identified clusters are then removed so that only the azimuthal shear clusters are

330 accumulated over time to produce rotation tracks. This eliminates the low background

331 azimuthal shear values not associated with circulations, making the tracks themselves

332 more isolated, as seen in Figure 8.

334 Typically, clusters of high azimuthal shear values associated with mesocyclones

335 persist through many time steps, whereas clusters associated with remaining non-

336 meteorological signatures typically only appear sporadically. To isolate the

337 meteorological rotation tracks, these non-meteorological shear clusters need to be

338 removed from the accumulated fields. Multiple Hypothesis Tracking (MHT) (Cox and

339 Hingorani 1996) techniques are used to isolate the continuous tracks of azimuthal shear

340 clusters associated with storms and remove the short-lived non-meteorological azimuthal

341 shear clusters.

342 MHT techniques have been used in the fields of video processing and military

343 target tracking for years and recently have been adopted by the meteorology community 
344 (e.g. Root et al. 2011). The technique attempts to associate objects, in this case azimuthal

345 shear clusters, throughout time. It is innovative as it considers time associations globally

346 and makes association decisions that can be deferred until additional information is

347 available. If the algorithm is not certain whether an existing track should be associated

348 with cluster A or cluster B in the current time step, for example, it can create two

349 hypotheses (see Figure 9). Both possibilities are then propagated forward in time to when

350 enough information should be available to determine which hypothesis most likely. The

351 clusters that do not meet the minimum longevity threshold (two time steps or roughly 10

352 minutes) are then retroactively pruned so that they are not admitted into the rotation track 353 fields.

354 An association cost matrix is constructed so an entry, $d_{i, j}$, indicates the cost of 355 matching cluster $i$ at one time step with cluster $j$ at the next time step. Each association

356 has a computed cost based on cluster sizes, ages, proximities to clusters from previous

357 time steps and other characteristics. The associations with the lowest cost are made.

358 Enumeration of all the hypothesis matrices to find the lowest costs can increase

359 exponentially with each time step, so a technique based on Murty (1968) is used to prune

360 the set to retain only the k-best associations at each time step. The algorithm is illustrated

361 in Figure 10. For more details and to see quantitative improvements in simulated fields

362 through the use of MHT, the reader is referred to Lakshmanan et al. (2012).

363 In an effort to remove any lingering non-meteorological clusters, a data value

364 distribution threshold was set. It was observed that the majority of clusters associated

365 with meteorological clusters exhibited unimodal distributions of azimuthal shear data

366 values with central tendencies while the non-meteorological clusters typically exhibited 
367 uniform distributions of very high azimuthal shear values (see examples in Figure 11).

368 To account for this, clusters are pruned if more than $80 \%$ of their pixel values are greater

369 than or equal to $0.02 \mathrm{~s}^{-1}$. This has only an incremental impact on the field itself since

370 most non-meteorological clusters are removed before this point.

$371 \quad$ Bands of high azimuthal shear values associated with linear meteorological

372 phenomena like outflow boundaries and bow echoes also appear in the rotation track

373 fields. In an effort to isolate the mesocyclone signatures from these bands of shear, all

374 clusters were fit to ellipses and their aspect ratios were calculated. After testing many

375 different thresholds, it was determined that size, data value distribution, and aspect ratio

376 information could not be successfully used to discriminate between mesocyclone clusters

377 and shear band clusters. Because of this, the band signatures remain in the rotation track

378 fields for now.

379 3. Results

380 The quality control techniques discussed in the methods section were developed

381 and tuned through testing on a variety of tornadic and nontornadic cases. The specific

382 impacts of each technique will now be discussed and demonstrated in this section using

383 cases that were not part of this training dataset.

384 a. New velocity dealiasing techniques

385 Prior to this study, the LED dealiasing technique (Eilts and Smith 1990), the

386 default method used for real-time processing of WSR-88D data, was used in the creation

387 of rotation tracks. Recently, it was found that using the vertical profile of horizontal wind

388 from the 20-km RUC point sounding at radar sites as estimates of the environmental wind 
389 in the algorithm helped to alleviate some of the dealiasing issues, though many still

390 persisted.

391 The two-dimensional dealiasing technique described by Jing and Weiner (1993)

392 was tested and appears to perform much better at properly dealiasing the velocity fields

393 due to the large reduction in radial spikes and non-meteorological velocity signatures.

394 Using the RUC wind profiles as environmental estimates made some additional

395 improvements as well. As seen by the representative example in Figure 12, the Jing and

396 Weiner technique dealiases correctly many of the areas that the LED technique did not.

397 Almost all of the noisy, high azimuthal shear values associated with dealiasing issues are

398 removed by using the Jing and Weiner technique, making the rotation tracks much easier

399 to interpret. A quantitative study to determine the best velocity dealiasing techniques is 400 ongoing.

\section{b. Range correction and reflectivity thresholds}

402 Whereas the azimuthal shear range correction does not make many visible

403 improvements to the rotation track fields, the azimuthal shear values in storm circulations 404 are more accurate estimates of the actual shear values. The ReflectivityQC threshold 405 below which all co-located shear data is removed is increased from $20 \mathrm{dBZ}$ to $40 \mathrm{dBZ}$. 406 This "stamping out" of azimuthal shear by the dilated ReflectivityQC field helps to 407 further isolate the azimuthal shear signatures associated with storms, as seen in Figure 13.

409 The rotation tracks are even further isolated from any background azimuthal shear 410 values through the use of hysteresis segmentation. The tracks are more isolated and easier 
411 to interpret (Fig. 14) since only the azimuthal shear clusters are accumulated over time

412 rather than the entire field, including the low background values.

414 After using hysteresis segmentation to form azimuthal shear clusters, the MHT

415 algorithm is used to isolate the persistent clusters associated with storm-scale circulations

416 from the non-meteorological clusters associated with any remaining poor velocity

417 dealiasing signatures. Figure 15 illustrates the removal of some small, leftover

418 circulations from the 27 April 2011 case by the MHT algorithm.

419 Figure 16 illustrates the differences between the original rotation track fields and

420 the rotation track fields after the quality control efforts were implemented on four recent

421 tornadic cases. Due to space constraints, only the overall improvements are shown.

422 Radial spikes, which were especially problematic in the 16 April 2011 case over Virginia

423 and North Carolina, were successfully removed. The low background azimuthal shear

424 values and nearly all azimuthal shear values not associated with storms are removed in all

425 four cases. Broad areas of non-mesocyclone shear still exist near some radar sites, but

426 overall a significant improvement is seen in the quality of the data and the ease of

427 interpretation.

428 To get a more quantitative idea of how MHT impacts rotation track fields, a

429 cluster-tracking algorithm described in Lakshmanan et al. (2003) was used to identify and

430 track the number of clusters in the 24 May 2011 event before and after implementing

431 MHT. Before MHT was implemented, 62 different clusters were identified and tracked,

432 whereas after MHT, only 41 clusters were identified and tracked in the eight hour case.

433 The low level rotation tracks associated with these post-MHT clusters compare well to 
434 reported tornado tracks in the several cases visually examined. Figure 17 shows how the

435 low level rotation track products associated with tornado damage paths from the 24 May

4362011 event across central Oklahoma compare to the EF-scale ratings.

\section{4. Conclusion}

$438 \quad$ NSSL rotation track products are valuable tools in disaster response situations.

439 They allow users to quickly assess both the spatial extent of mesocyclone circulations as

440 seen by radar over time and assess their relative intensities. While these products were

441 useful, a great deal of contamination initially was present due to poor velocity dealiasing,

442 ground clutter, radar test patterns and spurious shear values. These non-meteorological

443 signatures made the tracks nearly impossible to see in some extreme cases.

444 To mitigate these problems for both real-time use and for a multi-year rotation

445 track climatology as part of the MYRORSS project, quality control strategies were

446 developed and implemented. A two-dimensional velocity dealiasing technique using 20-

$447 \mathrm{~km}$ RUC wind data as input made large visual improvements in the quality of the initial

448 radial velocity field. An azimuthal shear range correction algorithm and some simple data

449 thresholds were added to the LLSD shear algorithm. Hysteresis segmentation was used to

450 isolate clusters of high azimuthal shear associated with mesocyclone circulations in each

451 time step of the two-dimensional maximum azimuthal shear fields and MHT techniques

452 were used to associate them throughout time. Any clusters that did not persist for at least

45310 minutes (2 time steps) or were comprised of an unrealistic distribution of high

454 azimuthal shear values were pruned. The remaining clusters were kept and used to create 455 the rotation track fields. 
456 While a few issues like the broad shear signatures around some radar sites remain,

457 overall the rotation track fields show a great deal of qualitative improvement after the

458 implementation of the quality control efforts. Whereas the tracks associated with the

459 mesocyclones initially were diluted by background noise and non-meteorological

460 signatures, they are now isolated and easier to interpret. Incorporating these

461 improvements, processing of the MYRORSS rotation track climatology should begin in

462 the near future.

$463 \quad$ Acknowledgments.

464 Funding for the authors was provided under NOAA-OU Cooperative Agreement

465 NA17RJ1227. The authors thank Kiel Ortega, Kevin Manross, John Cintineo, and

466 Jennifer Newman for all their help and advice. The authors also thank Gabe Garfield,

467 Kiel Ortega and Brandon Smith for allowing us to use their damage survey data for the

46824 May 2011 tornadoes.

469

470 


\section{References}

472 Bargen, D. W., and R. C. Brown, 1980: Interactive radar velocity unfolding. Preprints, 473 19th Conf. on Radar Meteorology, Miami, FL, Amer. Meteor. Soc., 278-283.

474

475 Benjamin, S. G., and Coauthors, 2004: An hourly assimilation-forecast cycle: The RUC. 476 Mon. Wea. Rev., 132, 495-518.

477

478 Bergen, W. R., and S. C. Albers, 1988: Two- and three-dimensional de-aliasing of 479 Doppler radar velocities. J. Atmos. Oceanic Technol., 5, 305-319.

481 Boren, T. A., J. R. Cruz, and D. S. Zrnić, 1986: An artificial intelligence approach to 482 Doppler weather radar velocity dealiasing., Proc. 23 rd Conf. on Radar Meteorology, 483 Snowmass, CO, Amer. Meteor. Soc., 107-110.

485 Brown, R. A., L. R. Lemon, and D. W. Burgess, 1978: Tornado detection by pulsed 486 Doppler radar. Mon. Wea. Rev., 106, 29-38.

488 Brown, R. A., V. T. Wood, and D. Sirmans, 2002: Improved tornado detection using 489 simulated and actual WSR-88D data with enhanced resolution. J. Atmos. Oceanic 490 Technol., 19, 1759-1771.

492 Cintineo, J., T. Smith, V. Lakshmanan, and S. Ansari, 2011: An automated system for 493 processing the Multi-Year Reanalysis of Remotely Sensed Storms (MYRORSS). 
494 Preprints, 27th Conf. on Interactive Information Processing Systems (IIPS), Seattle, WA, 495 Amer. Meteor. Soc., J9.3.

496

497 Clark, A. J., J. S. Kain, P. T. Marsh, J. Correia, Jr., M. Xue, and F. Kong, 2012:

498 Forecasting tornado path lengths using a 3-dimensional object algorithm applied to

499 convection-allowing forecasts. Wea. Forecasting, In Press.

500

501 Cox, I., and S. L. Hingorani, 1996: An efficient implementation of Reid's multiple

502 hypothesis tracking algorithm and its evaluation for the purpose of visual tracking. IEEE

503 Trans. Pattern Anal. Mach. Intell., 18, 138-150.

504

505 Crum, T. D., and R. K. Alberty, 1993: The WSR-88D and the WSR-88D Operational

506 Support Facility. Bull. Amer. Meteor. Soc., 74, 1669-1687.

507

508 Eilts, M. D., and S. D. Smith, 1990: Efficient dealiasing of Doppler velocities using local

509 environment constraints. J. Atmos. Oceanic Technol., 7, 118-128.

510

511 Elmore, K. M., E. D. Albo, R. K. Goodrich, and D. J. Peters, 1994: NASA/NCAR

512 airborne and ground-based wind shear studies. Final Report, contract no. NCC1-155, 343

513 pp.

514

515 Forbes, G. S., 1981: On the reliability of hook echoes as tornado indicators. Mon. Wea.

516 Rev., 109, 1457-1466. 
518 Fulton, R., D. Breidenback, D. Miller, and T. O’Bannon, 1998: The WSR-88D rainfall

519 algorithm. Wea. Forecasting, 13, 377-395.

520

521 Jain, A., 1989: Fundamentals of Digital Image Processing. Prentice Hall, 569 pp.

523 Jing, Z., and G. Wiener, 1993: Two-dimensional dealiasing of Doppler velocities. J.

524 Atmos. Oceanic Technol., 10, 798-808.

525

526 Kain, J. S., S. R. Dembek, S. J. Weiss, J. L. Case, J. J. Levit, R. A. Sobash, 2010:

527 Extracting unique information from high-resolution forecast models: Monitoring selected 528 fields and phenomena every time step. Wea. Forecasting, 25, 1536-1542.

530 Kessinger, C., S. Ellis, and J. Van Andel, 2003: The radar echo classifier: A fuzzy logic

531 algorithm for the WSR-88D. Pre-prints, Third Conf. on Artificial Applications to the

532 Environmental Sciences, Long Beach, CA, Amer. Meteor. Soc., CD-ROM, P1.6.

534 Lakshmanan, V., R. Rabin, and V. DeBrunner, 2003: Multiscale storm identification and 535 forecast. J. Atmos. Res., 367-380.

537 Lakshmanan, V., T. Smith, K. Hondl, G. J. Stumpf and A. Witt, 2006: A real-time, three538 dimensional, rapidly updating, heterogeneous radar merger technique for reflectivity, 539 velocity and derived products. Wea. Forecasting, 21, 802-823. 
541 Lakshmanan, V., A. Fritz, T. Smith, K. Hondl, and G. J. Stumpf, 2007a: An automated

542 technique to quality control radar reflectivity data. J. Appl. Meteor., 46, 288-305.

543

544 Lakshmanan, V., T. Smith, G. J. Stumpf, and K. Hondl, 2007b: The Warning Decision

545 Support System - Integrated Information. Wea. Forecasting, 22, 596-612.

547 Lakshmanan, V., J. Zhang, and K. Howard, 2010: A technique to censor biological

548 echoes in radar reflectivity data. J. Appl. Meteor., 49, 35-462.

550 Lakshmanan, V., 2012: Neighborhood and window operations. Automating the Analysis

551 of Spatial Grids: A Practical Guide to Data Mining Geospatial Images for Human \&

552 Environmental Applications, Springer, 129-171.

553

554 Lakshmanan, V., M. Miller, and T. Smith, 2012: Quality control of accumulated fields by 555 applying spatial and temporal constraints. J. Atmos. Ocean. Tech., Accepted. [Available 556 online at http://www.cimms.ou.edu/ lakshman/Papers/mhtqc.pdf.]

558 Liu, S., M. Xue, and Q. Xu, 2007: Using wavelet analysis to detect tornadoes from

559 Doppler radar radial-velocity observations. J. Atmos. Oceanic Technol., 24, 344-359.

560

561 Merritt, M. W., 1984: Automatic velocity dealiasing for real-time applications. Proc.

562 22nd Conf. on Radar Meteorology, Zurich, Amer. Meteor. Soc., 528-533. 
563 Mitchell, E. D., and K. L. Elmore, 1998: A technique for identifying regions of high

564 shear associated with mesocyclones and tornadic vortex signatures. Preprints, 14th

565 International Conference on Interactive Information and Processing Systems (IIPS) for

566 Meteorology, Oceanography, and Hydrology, Phoenix, AZ. Amer. Meteor. Soc., 312-

567315.

568

569 Mitchell, E. D., S. V. Vasiloff, G. J. Stumpf, A. Witt, M. D. Eilts, J. T. Johnson, K. W.

570 Thomas, 1998: The National Severe Storms Laboratory tornado detection algorithm.

571 Wea. Forecasting, 13, 352-366.

572

573 Murty, K. G., 1968: An algorithm for ranking all the assignments in order of increasing 574 cost. Operations Research, 16, 682-687.

575

576 Newman, J. F., V. Lakshmanan, P. L. Heinselman, M. B. Richman, and T. M. Smith, 577 2011: Range-correcting azimuthal shear in Doppler radar data. Wea. Forecasting, In 578 Press.

579

580 National Oceanic and Atmospheric Administration (NOAA) National Severe Storms

581 Laboratory (NSSL), cited 2011: NOAA technology helps American Red Cross respond

582 faster. [Available online at https://secure.nssl.noaa.gov/briefings/2011/06/noaa-

583 technology-helps-american-red-cross-respond-faster/] 
585 Ray, P. S., and C. Ziegler, 1977: De-aliasing first-moment Doppler estimates. J. Appl.

586 Meteor., 16, 563-564.

587

588 Root, B., M. Yeary, and T.-Y. Yu, 2011: Novel storm cell tracking with multiple

589 hypothesis tracking. Preprints, 27th Conf. on Interactive Information Processing Systems

590 (IIPS), Seattle, WA, Amer. Meteor. Soc., 8B.3.

591

592 Steiner, M., and J. A. Smith, 2002: Use of three-dimensional reflectivity structure for

593 automated detection and removal of nonprecipitating echoes in radar data. J. Atmos.

594 Oceanic Technol., 19, 673-686.

595

596 Stumpf, G. J., A. Witt, E. D. Mitchell, P. L. Spencer, J. T. Johnson, M. D. Eilts, K. W.

597 Thomas, and D. W. Burgess, 1998: The National Severe Storms Laboratory mesocyclone

598 detection algorithm for the WSR-88D. Wea. Forecasting, 13, 304-326.

599

600 Smith, T. M., and K. L. Elmore, 2004: The use of radial velocity derivatives to diagnose

601 rotation and divergence. Preprints, 11th Conf.on Aviation, Range and Aerospace,

602 Hyannis, MA, Amer. Meteor. Soc., P5.6.

603

604 Wood, V. T., and R. A. Brown, 1997: Effects of radar sampling on single-Doppler

605 velocity signatures of mesocyclones and tornadoes. Wea. Forecasting, 12, 928-938.

606

607 


\section{List of Figures}

609 Fig. 1. Low level (0-3 km AGL) rotation tracks from the 27 April 2011 tornado outbreak.

610 Swaths of high maximum azimuthal shear approximate the movement and strength of

611 mesocyclone circulations within the supercells that moved southwest to northeast across

612 the states of Alabama, Mississippi, and Tennessee between 16 UTC on 27 April and 00

613 UTC on 28 April.

614

615 FIG. 2. Low level (0-3 km AGL) rotation tracks across Virginia, North Carolina and

616 South Carolina from the 16 April 2011 tornado outbreak generated using the default

617 WSR-88D velocity dealiasing technique and without any quality control techniques. The

618 spikes of high azimuthal shear values are caused by poor velocity dealiasing along radials

619 and can make data interpretation difficult, if not impossible, in some areas.

620

621 FIG. 3. Radial velocity fields and the corresponding azimuthal shear fields from KDGX

622 on 27 April 2011 at 2151 UTC created using different dealiasing strategies. (a) Radial

623 velocity dealiased using the LED algorithm. (b) Azimuthal shear field associated with (a).

624 (c) Radial velocity dealiased using the LED algorithm with an environmental wind field

625 from an input 20-km RUC point sounding. (d) Azimuthal shear associated with (c). (e)

626 Radial velocity field dealiased using the Jing and Weiner (1993) technique with an

627 environmental wind field from an input 20-km RUC point sounding. (f) Azimuthal shear

628 field associated with (e).

629

630 
631 FIG. 4. Flow chart showing how rotation track products are created. Grey boxes with

632 dashed lines represent algorithms and white boxes with solid lines represent data fields.

633

634 FIG. 5. (a) Low level (0-3 km AGL) rotation track field from the 2 March 2012 outbreak

635 from the KVWX radar site before data removal near the radar site. Note the high values

636 of azimuthal shear surrounding the radar site at the center of the image. (b) The same

637 rotation track field after the removal of data within a 5-km radius of the radar site. This

638 removal will only be performed for the climatology, not in real-time.

639

640 FIG. 6. (a) Reflectivity, (b) ReflectivityQC, (c) radial velocity, and (d) azimuthal shear

641 fields associated with a supercell over central Alabama on 27 April 2011 at 2107 UTC.

642

643 FIG. 7. A schematic showing how hysteresis segmentation works. The first and last

644 peaks of azimuthal shear values will be associated with clusters, while the middle peak

645 will not be associated with a cluster because it contains no values above the higher data

646 threshold.

647

648 FIG. 8. Low level (0-3 km AGL) rotation tracks associated with the tornadic supercells

649 that moved across Mississippi and Alabama between 16 UTC on 27 April 2011 and 00

650 UTC on 28 April 2011. (a) Rotation tracks before hysteresis segmentation. (b) Rotation

651 tracks after hysteresis segmentation is used to threshold the field.

652 
653 FIG. 9. Example of how the MHT algorithm tracks clusters and generates hypotheses.

654 Solid shapes represent the position of azimuthal shear clusters at the given time step.

655 Solid arrows show the movement of clusters between time steps. Dotted shapes show the

656 projected locations of the azimuthal shear clusters in the next time steps. Dashed arrows

657 show the projected movement of the clusters between time steps. Shape A shows the

658 projected location of the original cluster at time t. Shape B represents the actual location

659 of the original cluster at time t. The algorithm generates two hypotheses for time $t+1$ (C

660 and $\mathrm{D}$ ). If the location of $\mathrm{B}$ is an error, then at time $\mathrm{t}+1$ the cluster should move to

661 position D. If the location of B is not an error but a change in motion, then B should

662 move to location $\mathrm{C}$ at time $\mathrm{t}+1$. At time $\mathrm{t}+1$, the discovery of the target at either $\mathrm{C}$ or $\mathrm{D}$

663 will confirm one hypothesis and disprove the other. The disproved cluster is deleted and

664 the confirmed one continues on to the next time step. In this case, given the strange shape

665 and size of B, it is likely that this cluster is associated with a non-meteorological shear

666 signature and will not persist in time $\mathrm{t}+1$.

667

668 FIG. 10. Multiple hypothesis tracking flow chart adapted from Root et al. (2011).

669

670 FIG. 11. An example of a cluster of high azimuthal shear values associated with a

671 mesocyclone circulation is shown in (a) and the histogram of its data values after

672 hysteresis segmentation is shown in (b). An example of a cluster of high azimuthal shear

673 values associated with a non-meteorological artifact is shown in (c) and the histogram of

674 its data values after hysteresis segmentation is shown in (d). Note the nearly uniform

675 distribution of very high values. 
677 FIG. 12. Low level (0-3 km AGL) rotation tracks associated the 27 April 2011 tornado

678 outbreak across Mississippi and Alabama produced using different velocity dealiasing

679 techniques, no thresholds and no MHT. (a) Tracks made using velocity dealiased with

680 the LED algorithm. (b) Tracks made using velocity dealiased with the LED algorithm

681 with 20-km RUC input sounding. (c) Tracks made using velocity dealiased with the Jing

682 and Weiner (1993) technique. (d) Tracks made using velocity dealiased with the Jing and

683 Weiner (1993) technique with 20-km RUC input sounding.

684

685 FIG. 13. Low level (0-3 km AGL) rotation tracks from the 27 April 2011 tornado

686 outbreak. (a) Tracks created using the Jing and Weiner (1993) velocity dealiasing

687 method with the input 20-km RUC sounding, without the azimuthal shear range

688 correction and without the increased ReflectivityQC threshold. (b) Tracks created using

689 the same velocity dealiasing method as in (a), but with the azimuthal shear range

690 correction and the increased ReflectivityQC threshold. Note that the circled area of high

691 azimuthal shear values associated with dealiasing errors over central Tennessee is much

692 less prominent in (b).

693

694 FIG. 14. Low level (0-3 km AGL) rotation track from the 27 April 2011 tornado

695 outbreak. (a) Tracks created using the Jing and Weiner (1993) velocity dealiasing

696 method with the input 20-km RUC sounding, with range correction, increased

697 ReflectivityQC thresholds and without hysteresis segmentation. (b) The same tracks as

698 (a) but with hysteresis segmentation implemented. 
700 FIG. 15. Low level (0-3 km AGL) rotation tracks across Mississippi and Alabama from

701 the 27 April 2011 tornado outbreak. (a) Tracks created using the Jing and Weiner (1993)

702 velocity dealiasing method with the input 20-km RUC sounding, with range correction,

703 increased ReflectivityQC thresholds, hysteresis segmentation and without MHT. (b) The

704 same tracks as (a) but with MHT. Note the several small clusters in west-central

705 Mississippi are removed.

706

707 FIG. 16. The impacts of the quality control efforts on low level (0-3 km AGL) rotation

708 track products associated with four recent tornado events: the 27 April 2011 event before

709 (a) and after (b) quality control, the 16 April 2011 event before (c) and after (d) quality

710 control, the 24 May 2011 event before (e) and after (f) quality control, and the 2 March

7112012 event before (g) and after (h) quality control.

712

713 FIG. 17. (a) Low level (0-3 km AGL) rotation tracks over a 145-minute period associated

714 with the 24 May 2011 tornado outbreak across central Oklahoma. (b) Plotted tornado EF-

715 scale ratings associated with tornadoes that occurred during that same time period as (a).

716 Survey data provided courtesy of Kiel Ortega, Brandon Smith, and Gabe Garfield. 


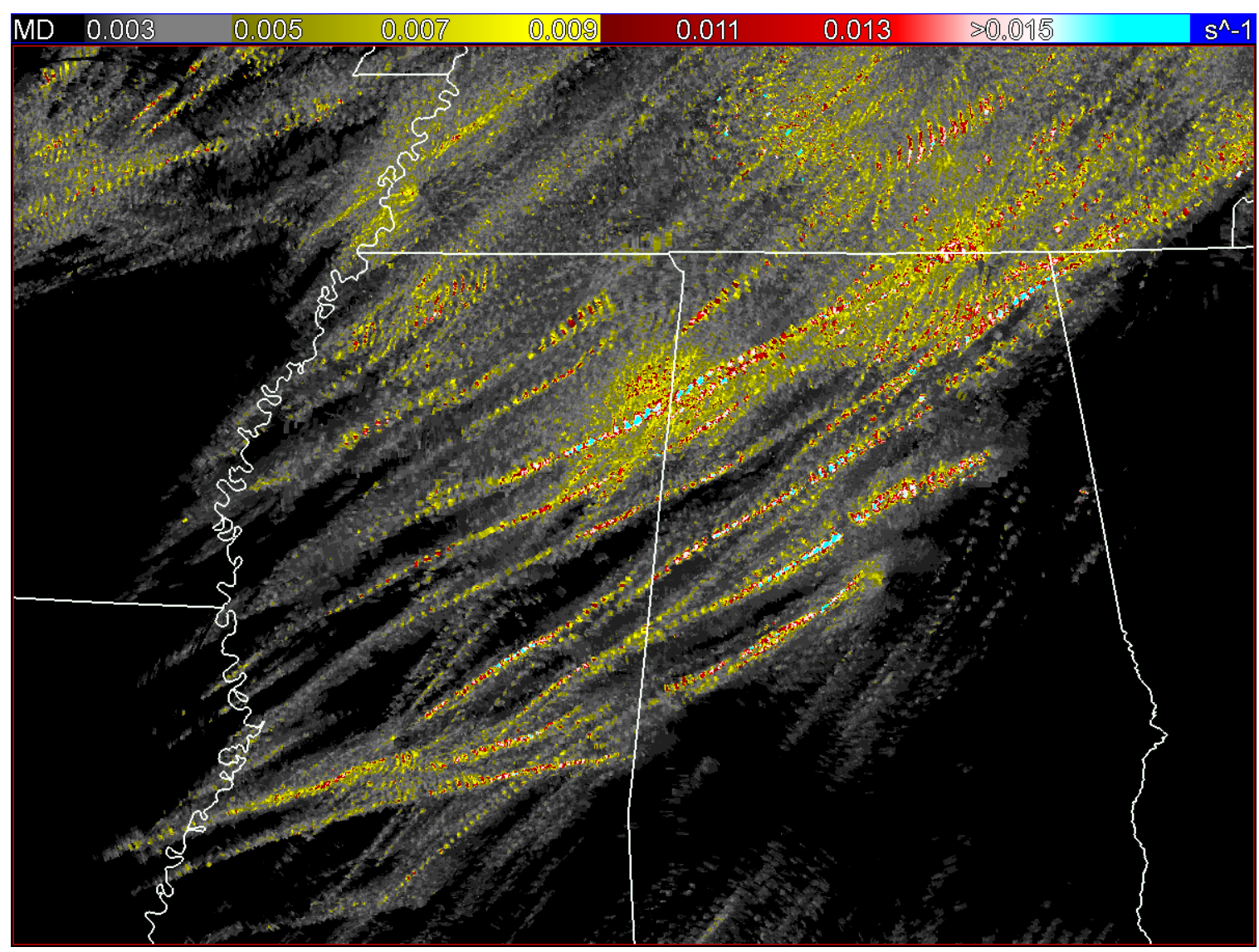

720 FIG. 1. Low level (0-3 km AGL) rotation tracks from the 27 April 2011 tornado outbreak.

721 Swaths of high maximum azimuthal shear approximate the movement and strength of

722 mesocyclone circulations within the supercells that moved southwest to northeast across

723 the states of Alabama, Mississippi, and Tennessee between 16 UTC on 27 April and 00

724 UTC on 28 April. 


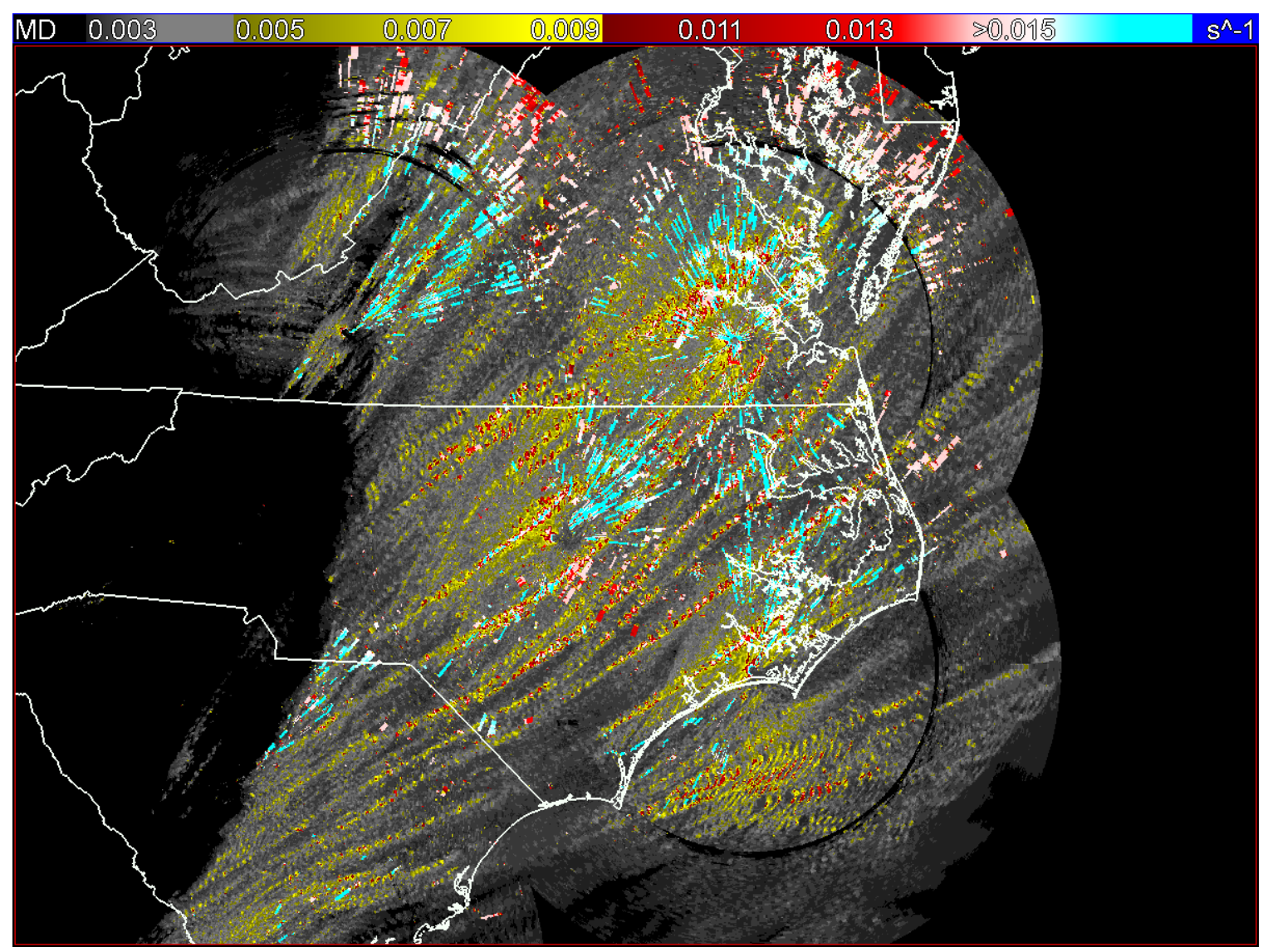

727 FIG. 2. Low level (0-3 km AGL) rotation tracks across Virginia, North Carolina and

728 South Carolina from the 16 April 2011 tornado outbreak generated using the default

729 WSR-88D velocity dealiasing technique and without any quality control techniques. The

730 spikes of high azimuthal shear values are caused by poor velocity dealiasing along radials

731 and can make data interpretation difficult, if not impossible, in some areas. 

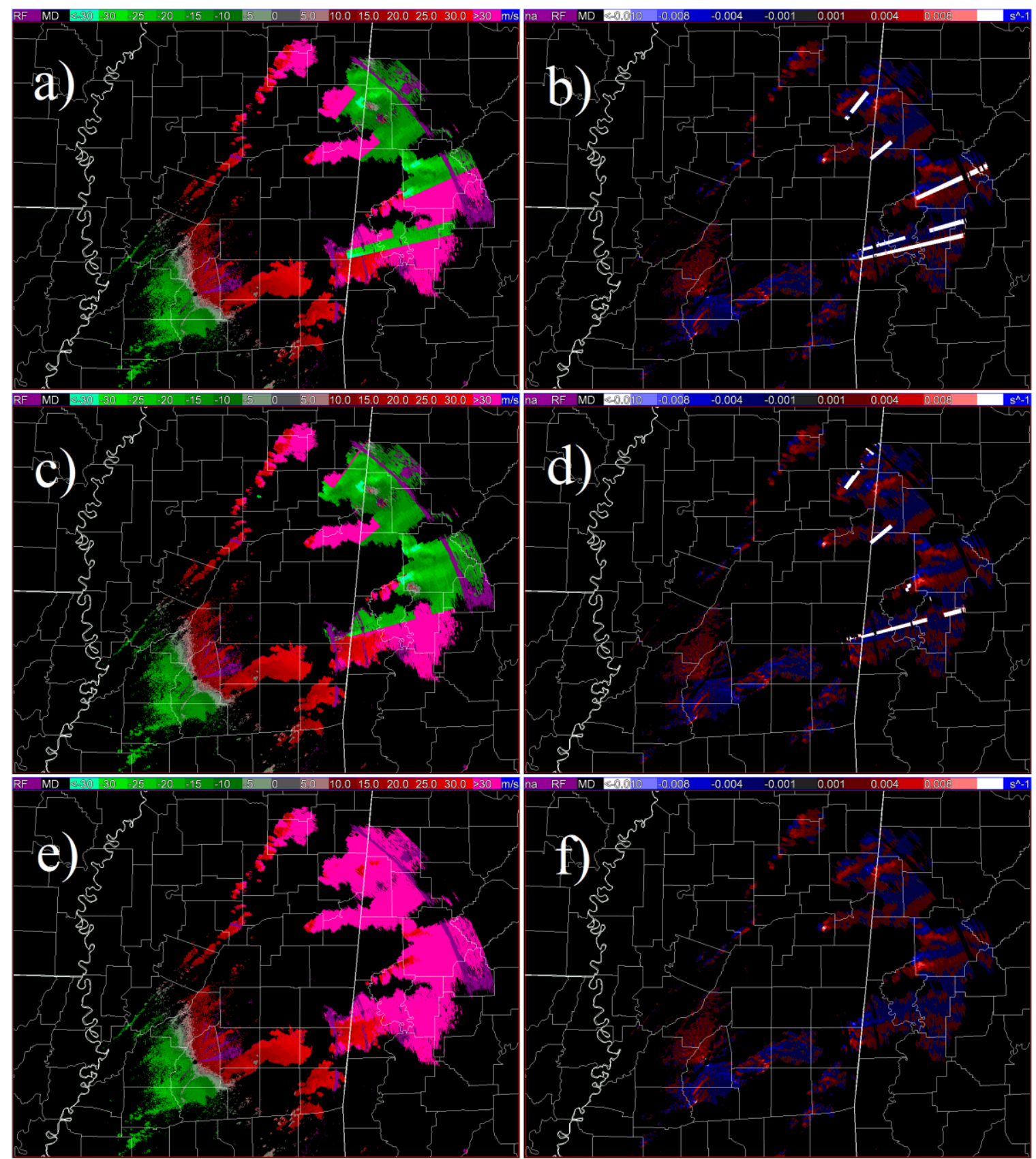

FIG. 3. Radial velocity fields and the corresponding azimuthal shear fields from KDGX

735 on 27 April 2011 at 2151 UTC created using different dealiasing strategies. (a) Radial

736 velocity dealiased using the LED algorithm. (b) Azimuthal shear field associated with (a).

737 (c) Radial velocity dealiased using the LED algorithm with an environmental wind field

738 from an input 20-km RUC point sounding. (d) Azimuthal shear associated with (c). (e)

739 Radial velocity field dealiased using the Jing and Weiner (1993) technique with an

740 environmental wind field from an input 20-km RUC point sounding. (f) Azimuthal shear

741 field associated with (e). 


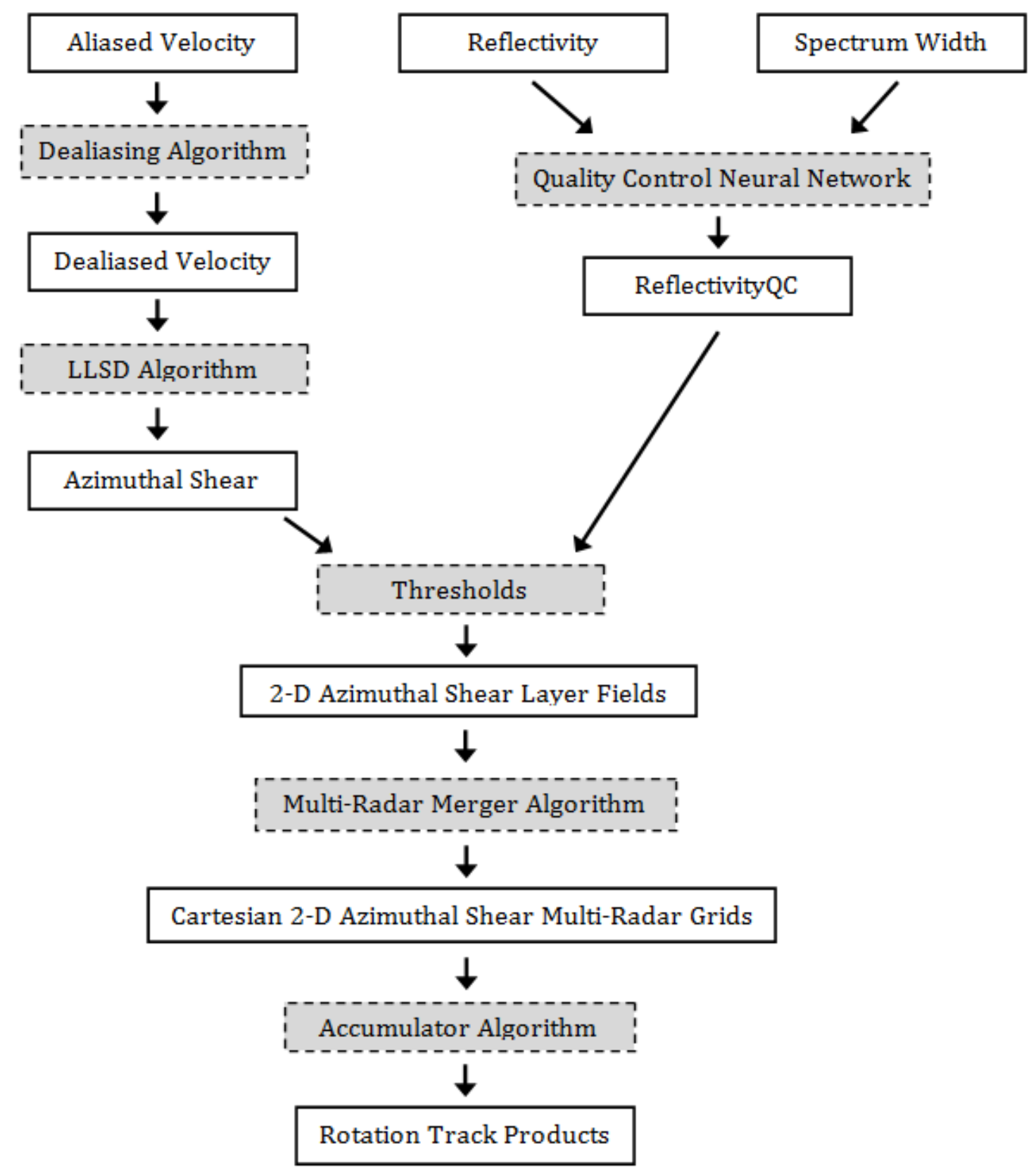

744 FIG. 4. Flow chart showing how rotation track products are created. Grey boxes with 745 dashed lines represent algorithms and white boxes with solid lines represent data fields. 

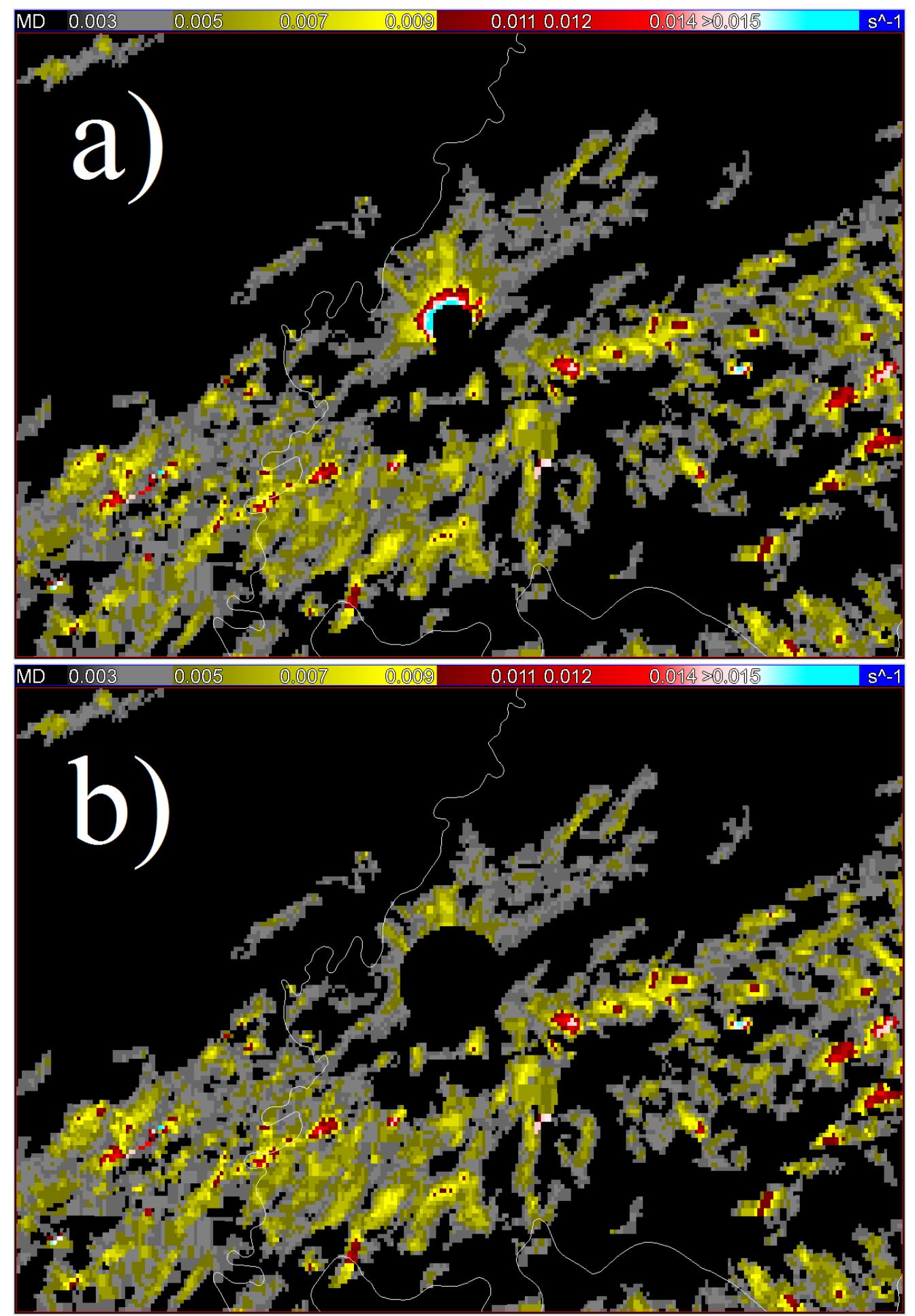

FIG. 5. (a) Low level (0-3 km AGL) rotation track field from the 2 March 2012

749 outbreak from the KVWX radar site before data removal near the radar site. Note the

750 high values of azimuthal shear surrounding the radar site at the center of the image. (b)

751 The same rotation track field after the removal of data within a 5-km radius of the radar

752 site. This removal will only be performed for the climatology, not in real-time. 


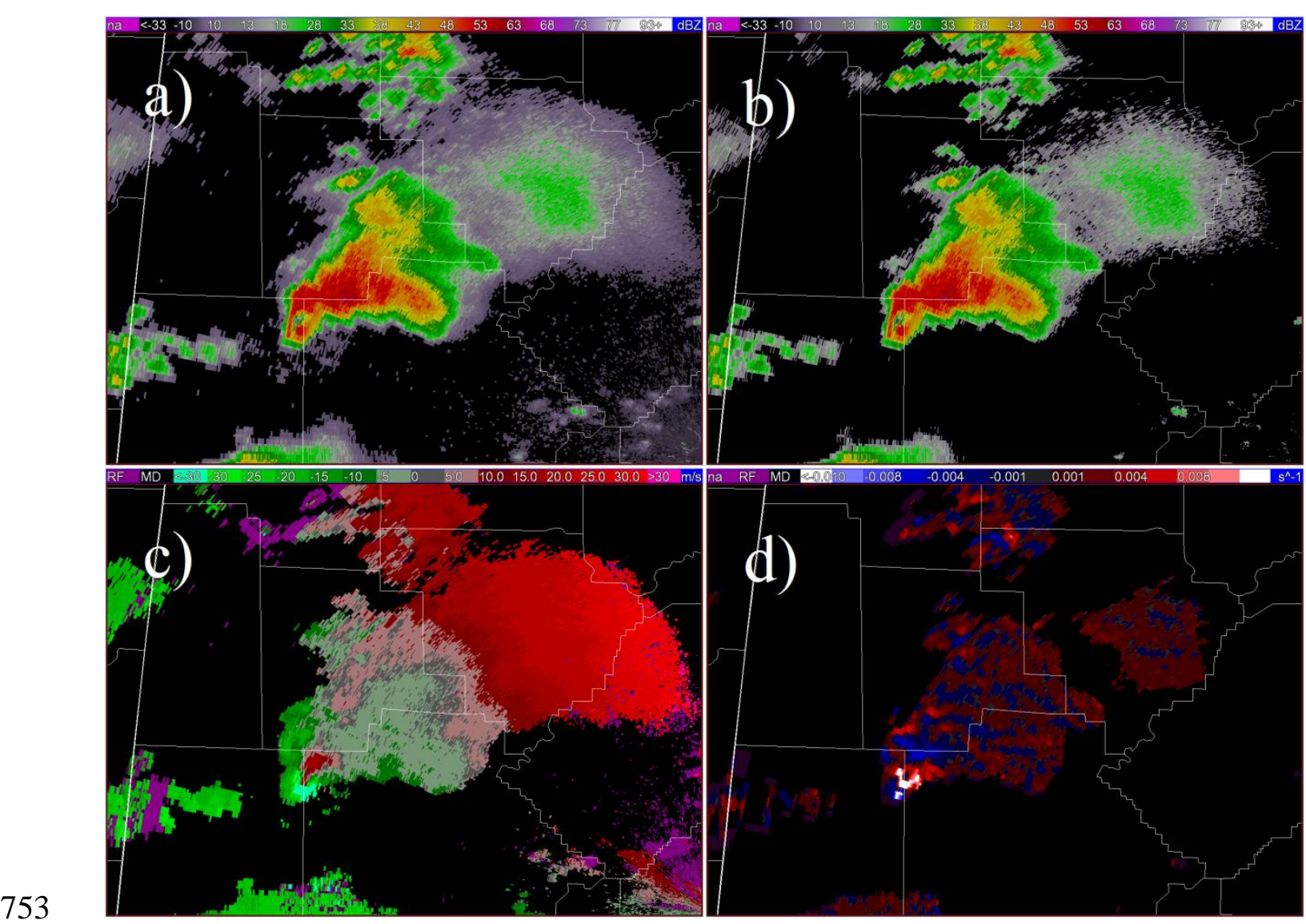

754 FIG. 6. (a) Reflectivity, (b) ReflectivityQC, (c) radial velocity, and (d) azimuthal shear

755 fields associated with a supercell over central Alabama on 27 April 2011 at 2107 UTC.

756

757

758

759

760

761

762

763

764 


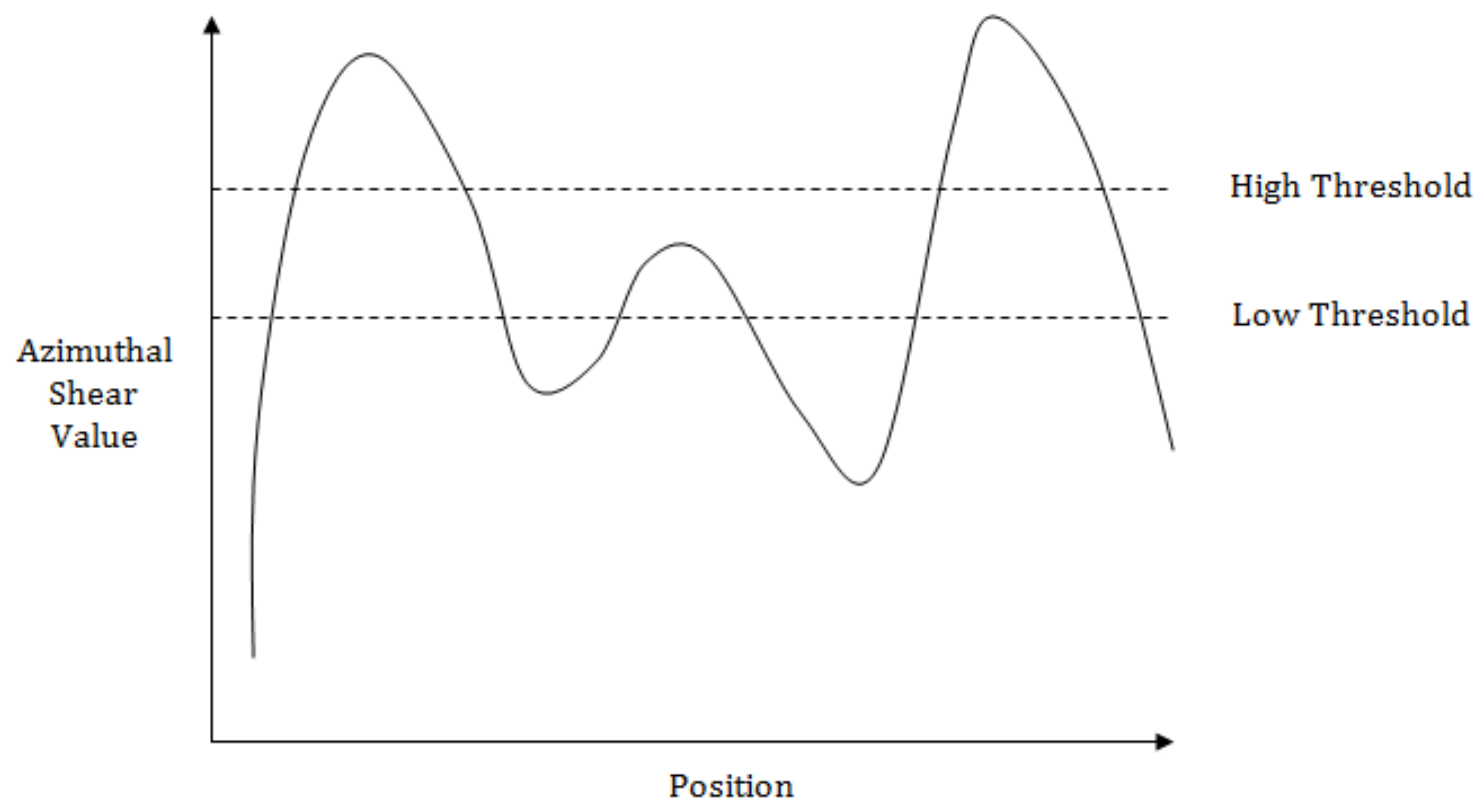

767 FIG. 7. A schematic showing how hysteresis segmentation works. The first and last peaks 768 of azimuthal shear values will be associated with clusters, while the middle peak will not 769 be associated with a cluster because it contains no values above the higher data threshold. 

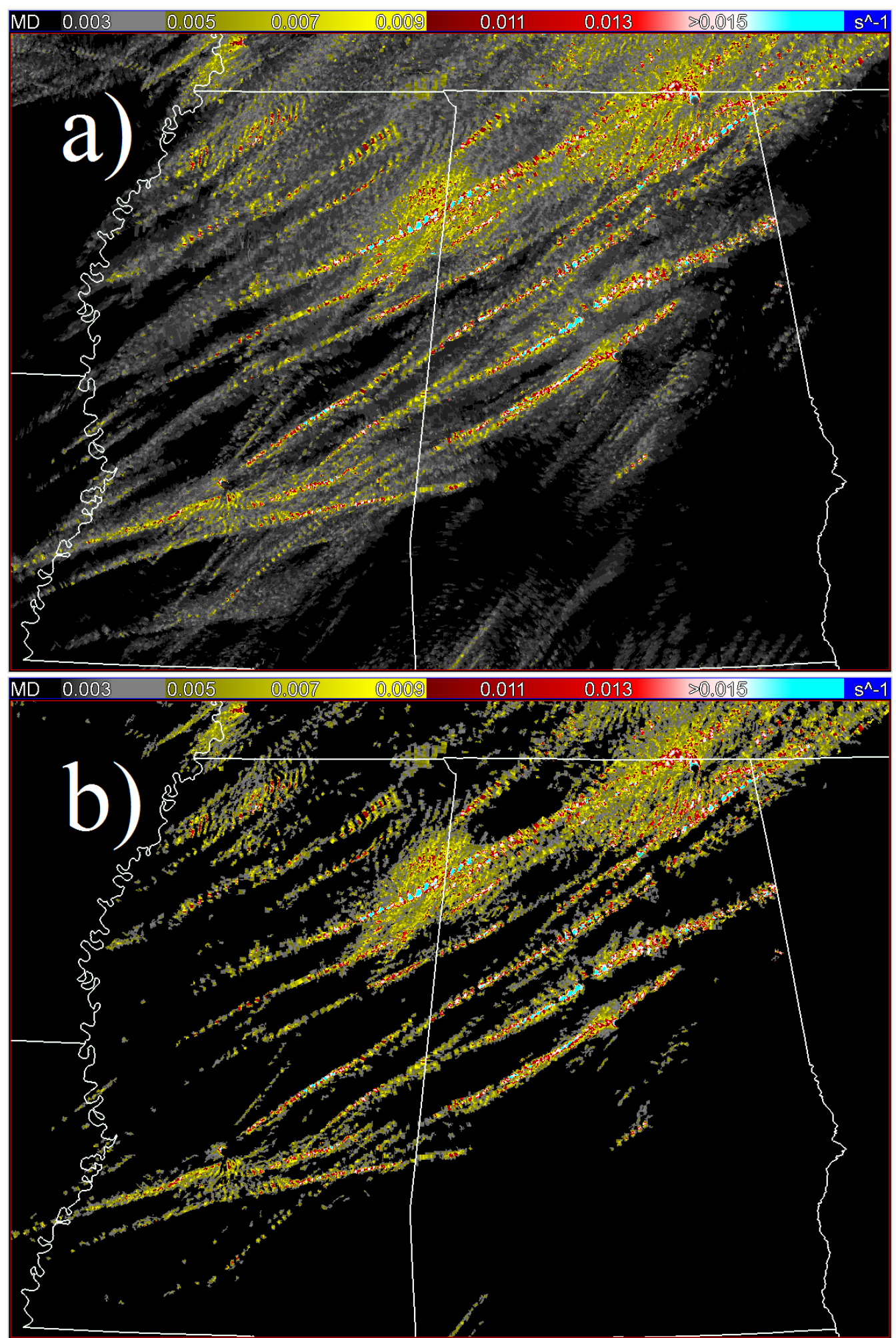

775 FIG. 8. Low level (0-3 km AGL) rotation tracks associated with the tornadic supercells

776 that moved across Mississippi and Alabama between 16 UTC on 27 April 2011 and 00

777 UTC on 28 April 2011. (a) Rotation tracks before hysteresis segmentation. (b) Rotation

778 tracks after hysteresis segmentation is used to threshold the field. 


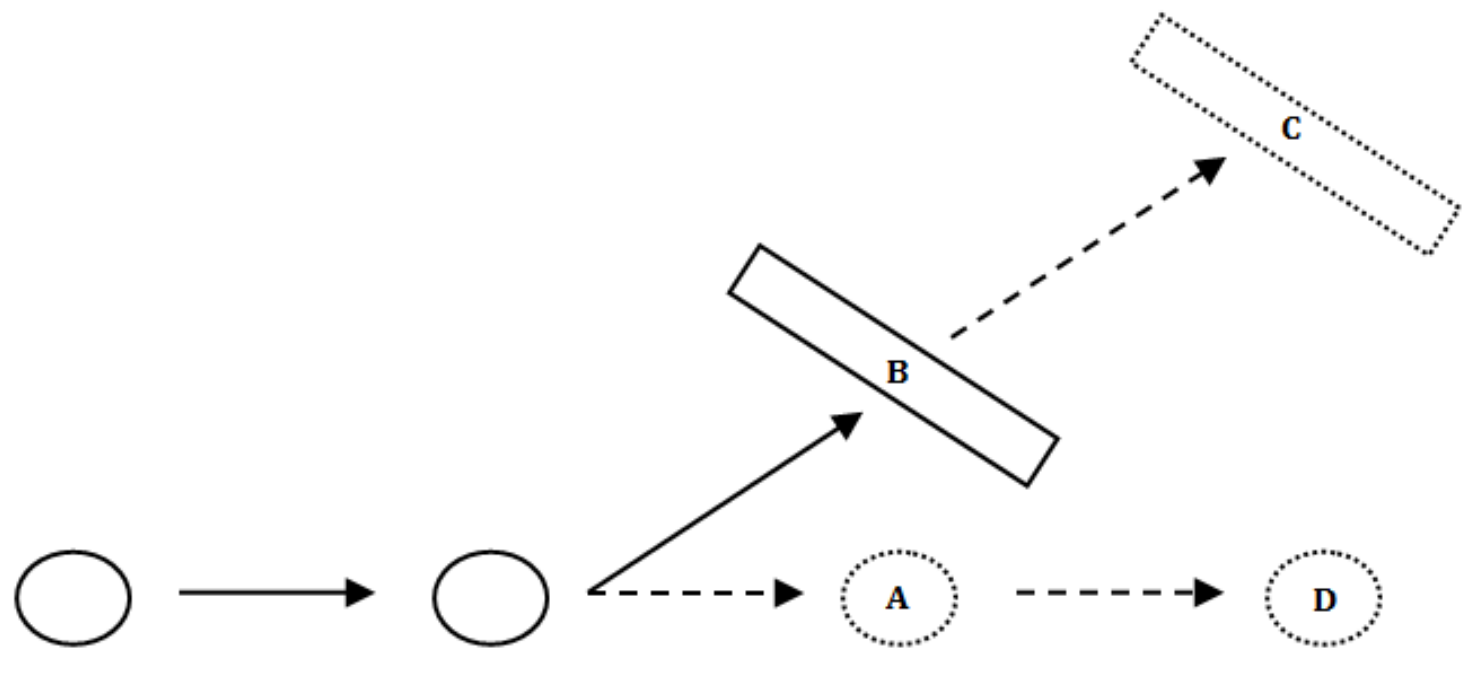

Time $\mathrm{t}-2$

Time $\mathrm{t}-1$

Time $t$

Time $t+1$

781 FIG. 9. Example of how the MHT algorithm tracks clusters and generates hypotheses.

782 Solid shapes represent the position of azimuthal shear clusters at the given time step.

783 Solid arrows show the movement of clusters between time steps. Dotted shapes show the

784 projected locations of the azimuthal shear clusters in the next time steps. Dashed arrows

785 show the projected movement of the clusters between time steps. Shape A shows the

786 projected location of the original cluster at time t. Shape B represents the actual location

787 of the original cluster at time $\mathrm{t}$. The algorithm generates two hypotheses for time $\mathrm{t}+1$ (C

788 and $\mathrm{D}$ ). If the location of $\mathrm{B}$ is an error, then at time $\mathrm{t}+1$ the cluster should move to

789 position $\mathrm{D}$. If the location of $\mathrm{B}$ is not an error but a change in motion, then $\mathrm{B}$ should

790 move to location $\mathrm{C}$ at time $\mathrm{t}+1$. At time $\mathrm{t}+1$, the discovery of the target at either $\mathrm{C}$ or $\mathrm{D}$

791 will confirm one hypothesis and disprove the other. The disproved cluster is deleted and

792 the confirmed one continues on to the next time step. In this case, given the strange shape

793 and size of $\mathrm{B}$, it is likely that this cluster is associated with a non-meteorological shear

794 signature and will not persist in time $\mathrm{t}+1$.

795

796 


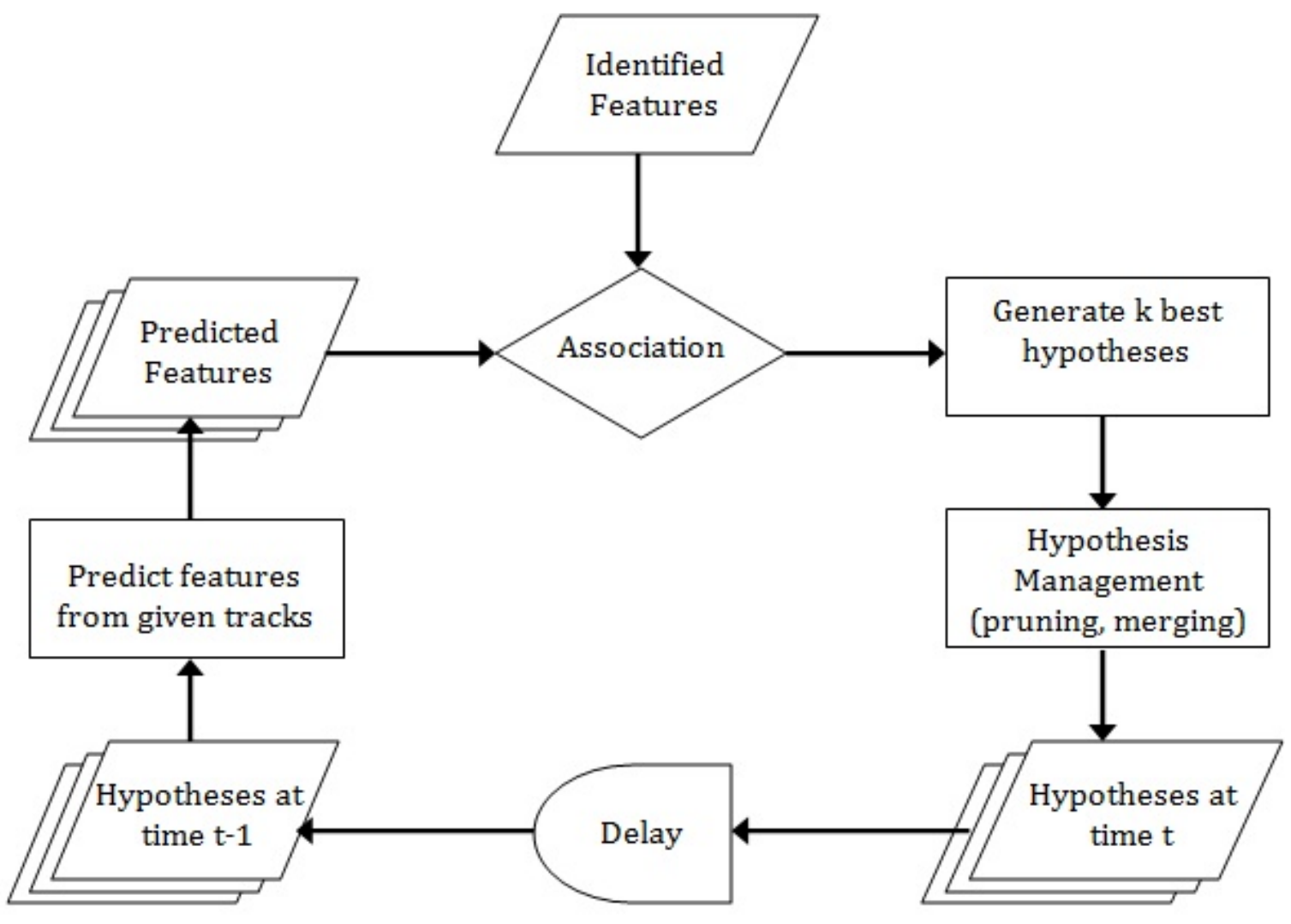

798

799 FIG. 10. Multiple hypothesis tracking flow chart adapted from Root et al. (2011).

800 

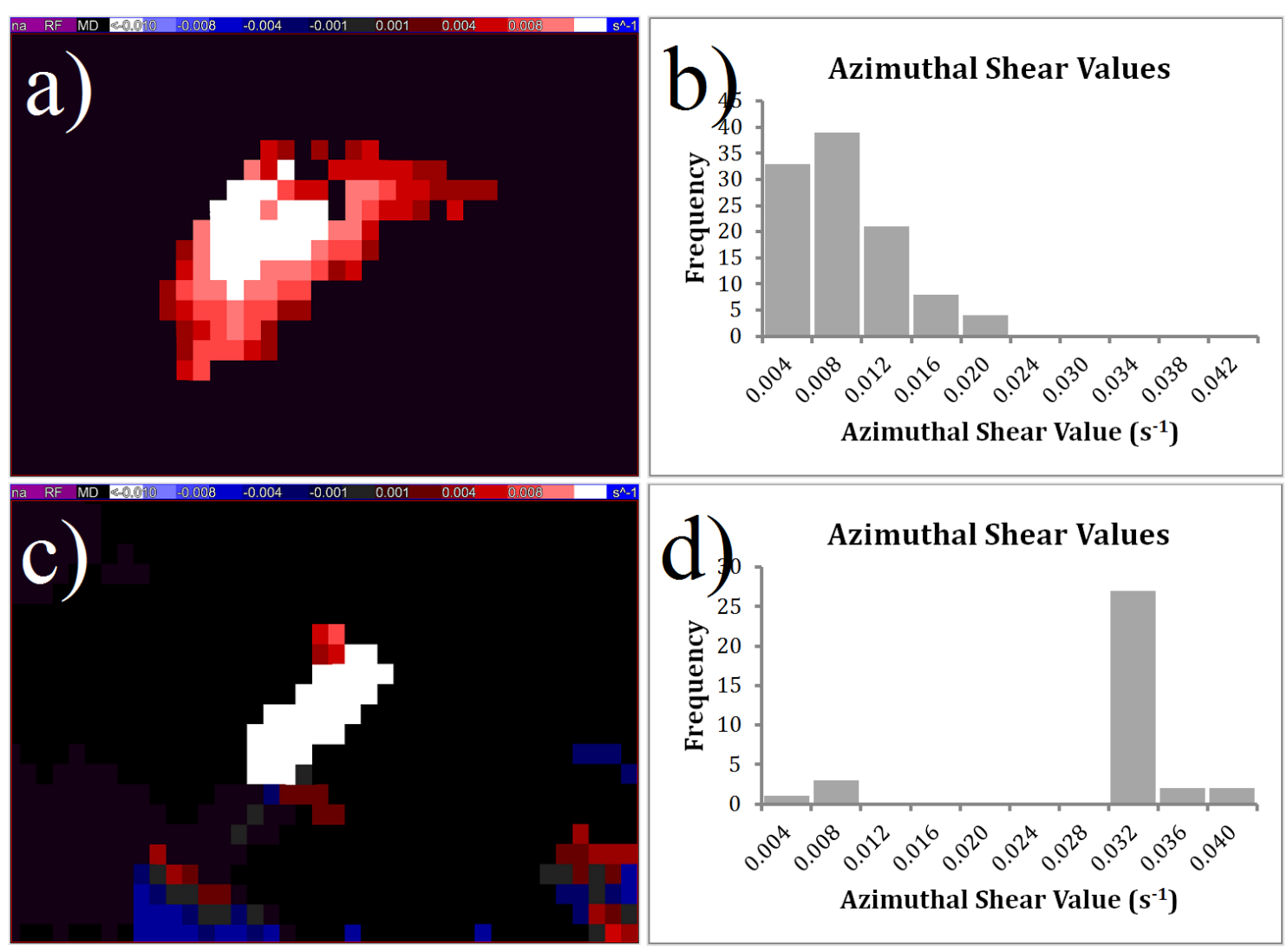

802 FIG. 11. An example of a cluster of high azimuthal shear values associated with a

803 mesocyclone circulation is shown in (a) and the histogram of its data values after

804 hysteresis segmentation is shown in (b). An example of a cluster of high azimuthal shear

805 values associated with a non-meteorological artifact is shown in (c) and the histogram of

806 its data values after hysteresis segmentation is shown in (d). Note the nearly uniform

807 distribution of very high values.

808

809

810 

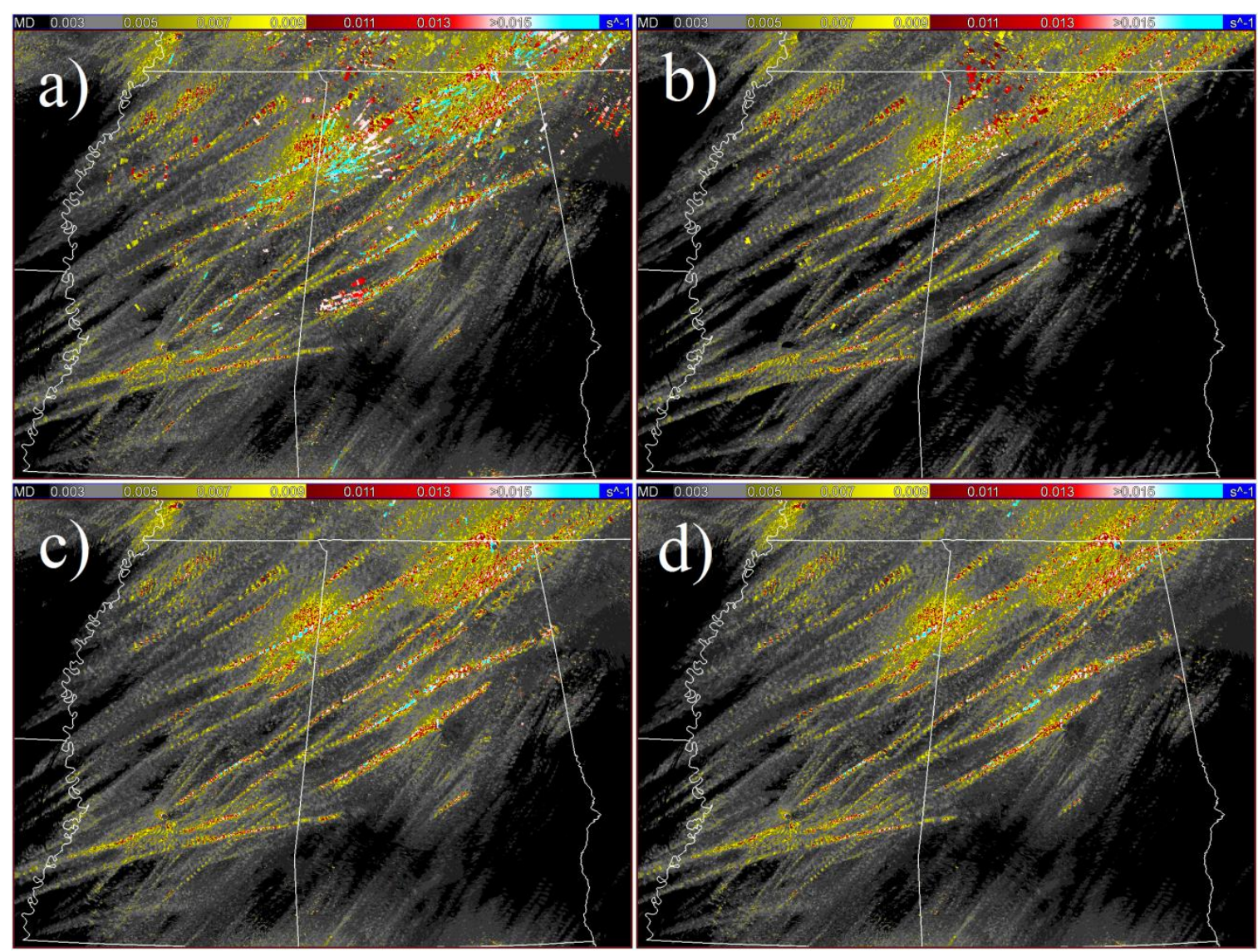

FIG. 12. Low level (0-3 km AGL) rotation tracks associated the 27 April 2011 tornado

813 outbreak across Mississippi and Alabama produced using different velocity dealiasing 814 techniques, no thresholds and no MHT. (a) Tracks made using velocity dealiased with

815 the LED algorithm. (b) Tracks made using velocity dealiased with the LED algorithm 816 with 20-km RUC input sounding. (c) Tracks made using velocity dealiased with the Jing 817 and Weiner (1993) technique. (d) Tracks made using velocity dealiased with the Jing and 818 Weiner (1993) technique with 20-km RUC input sounding. 

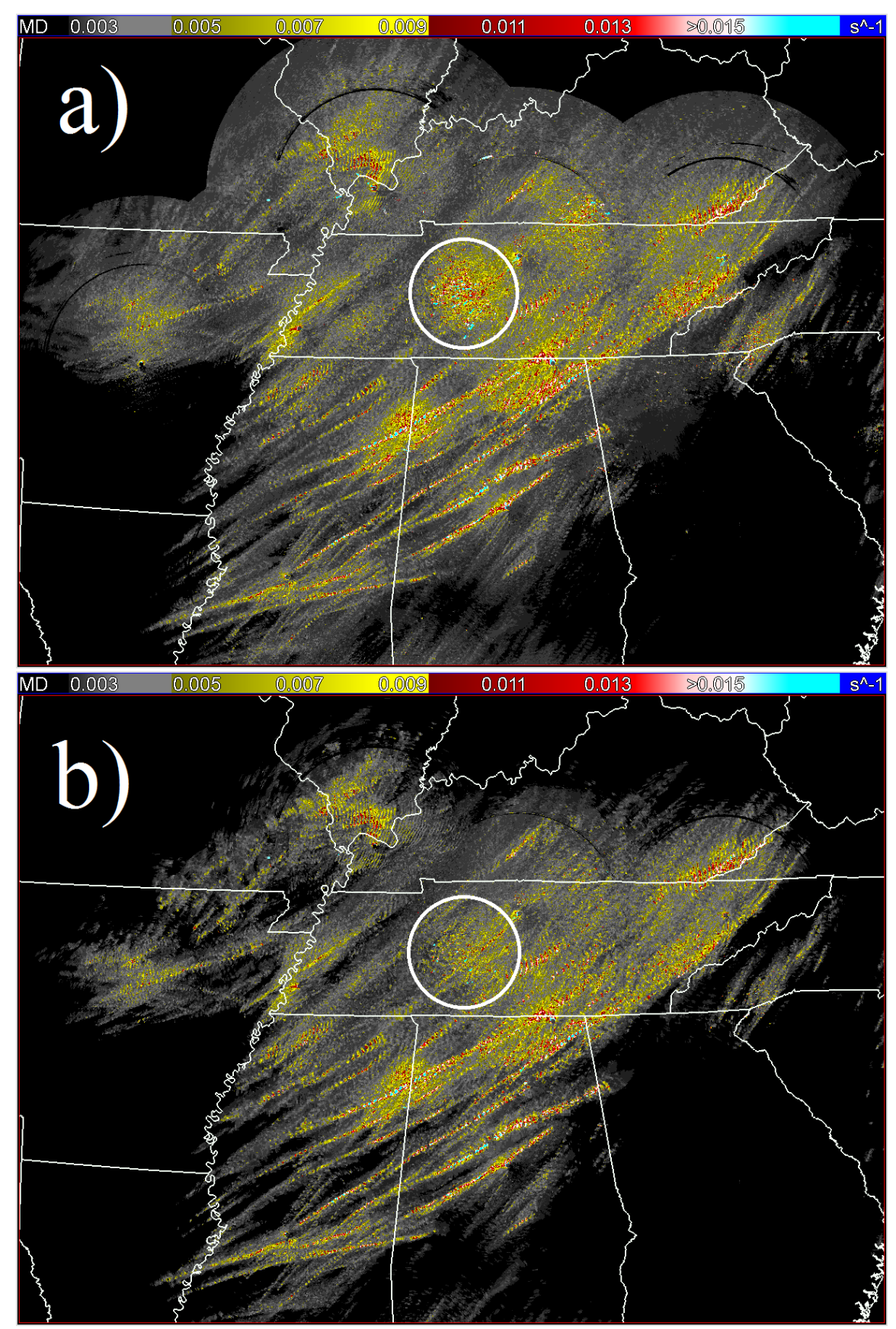

823 FIG. 13. Low level (0-3 km AGL) rotation tracks from the 27 April 2011 tornado

824 outbreak. (a) Tracks created using the Jing and Weiner (1993) velocity dealiasing

825 method with the input 20-km RUC sounding, without the azimuthal shear range

826 correction and without the increased ReflectivityQC threshold. (b) Tracks created using

827 the same velocity dealiasing method as in (a), but with the azimuthal shear range

828 correction and the increased ReflectivityQC threshold. Note that the circled area of high

829 azimuthal shear values associated with dealiasing errors over central Tennessee is much

830 less prominent in (b). 

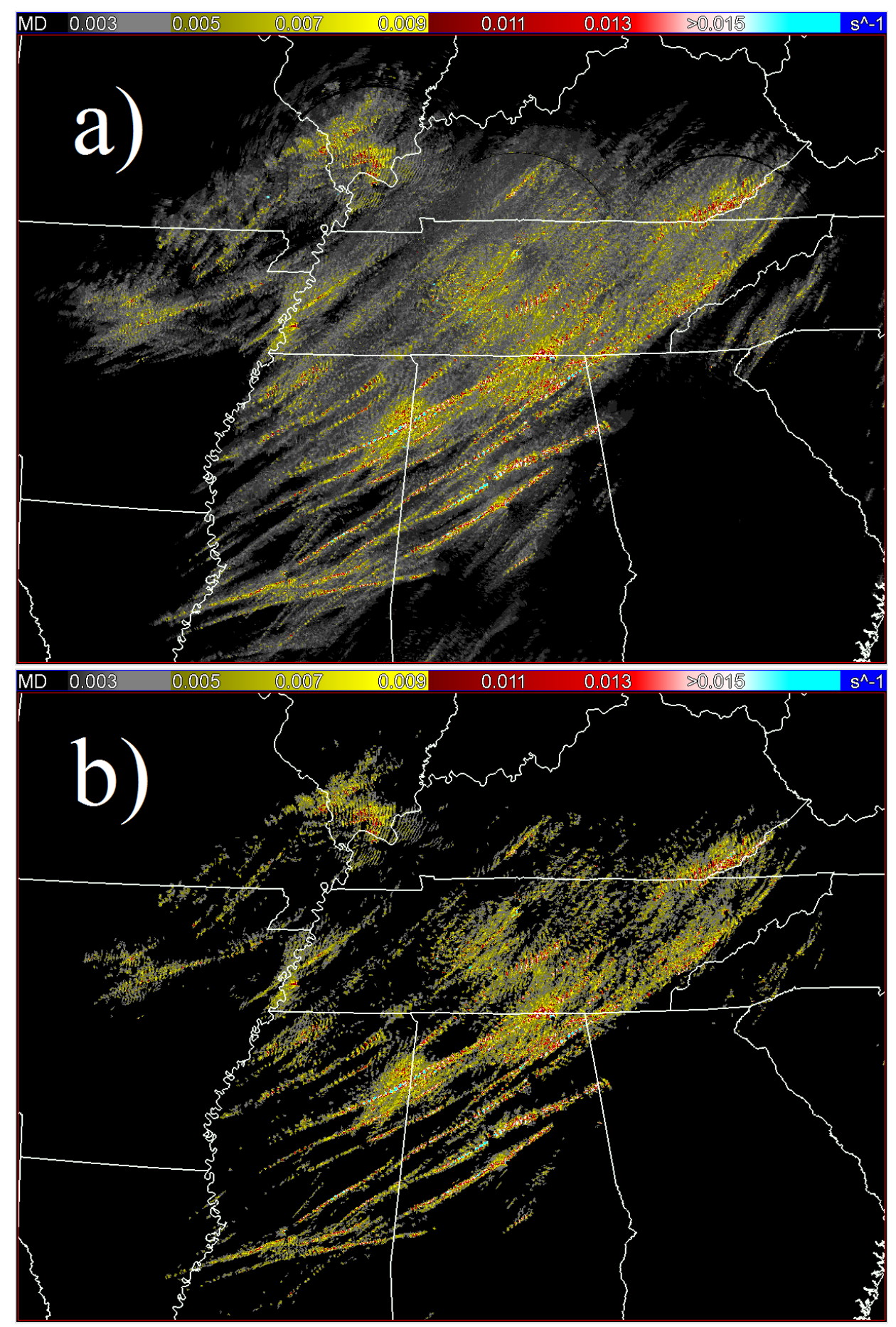

833 FIG. 14. Low level (0-3 km AGL) rotation track from the 27 April 2011 tornado

834 outbreak. (a) Tracks created using the Jing and Weiner (1993) velocity dealiasing 835 method with the input 20-km RUC sounding, with range correction, increased

836 ReflectivityQC thresholds and without hysteresis segmentation. (b) The same tracks as 837 (a) but with hysteresis segmentation implemented. 


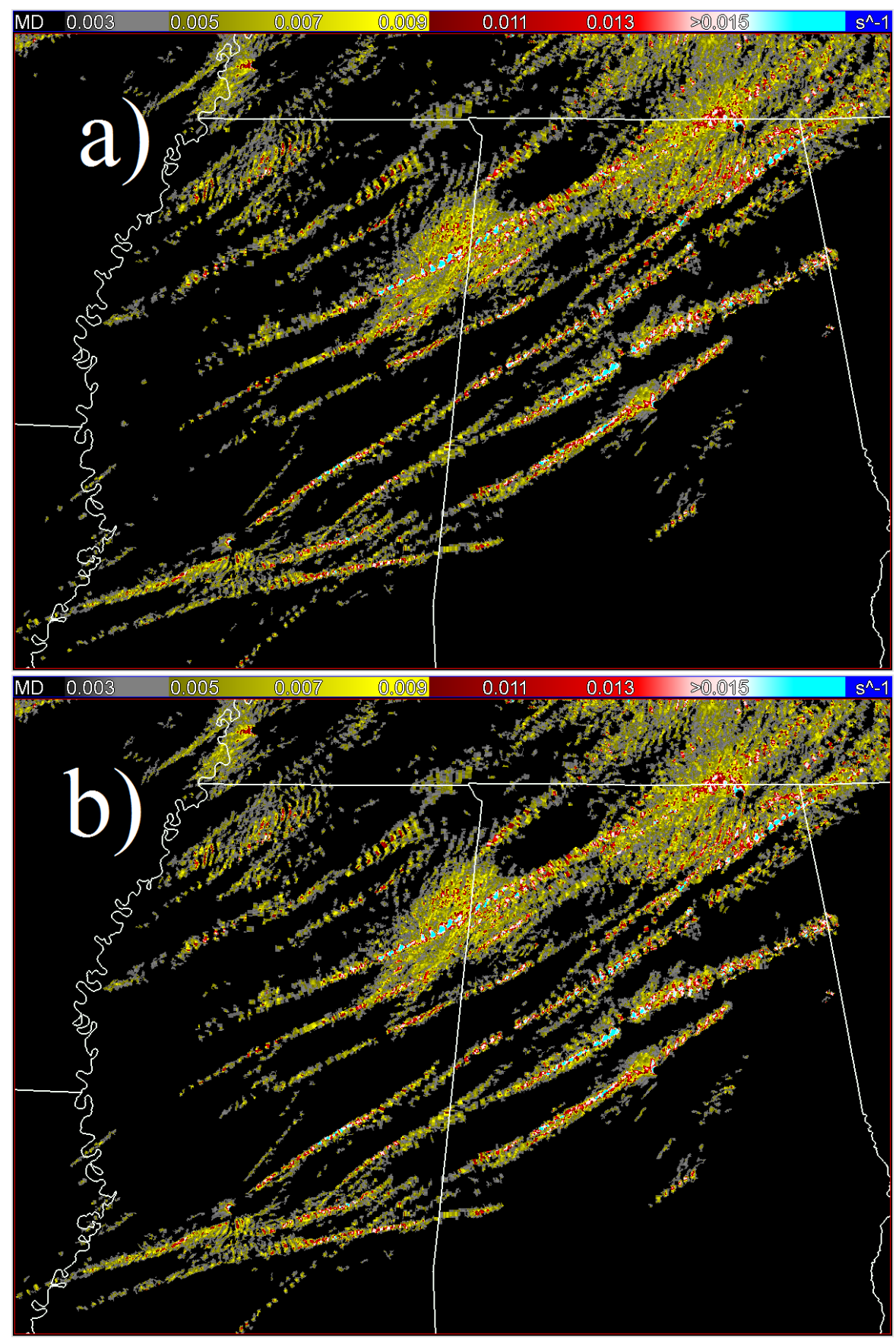

841 FIG. 15. Low level (0-3km AGL) rotation tracks across Mississippi and Alabama from 842 the 27 April 2011 tornado outbreak. (a) Tracks created using the Jing and Weiner (1993) 843 velocity dealiasing method with the input 20-km RUC sounding, with range correction, 844 increased ReflectivityQC thresholds, hysteresis segmentation and without MHT. (b) The 845 same tracks as (a) but with MHT. Note the several small clusters in west-central 846 Mississippi are removed. 

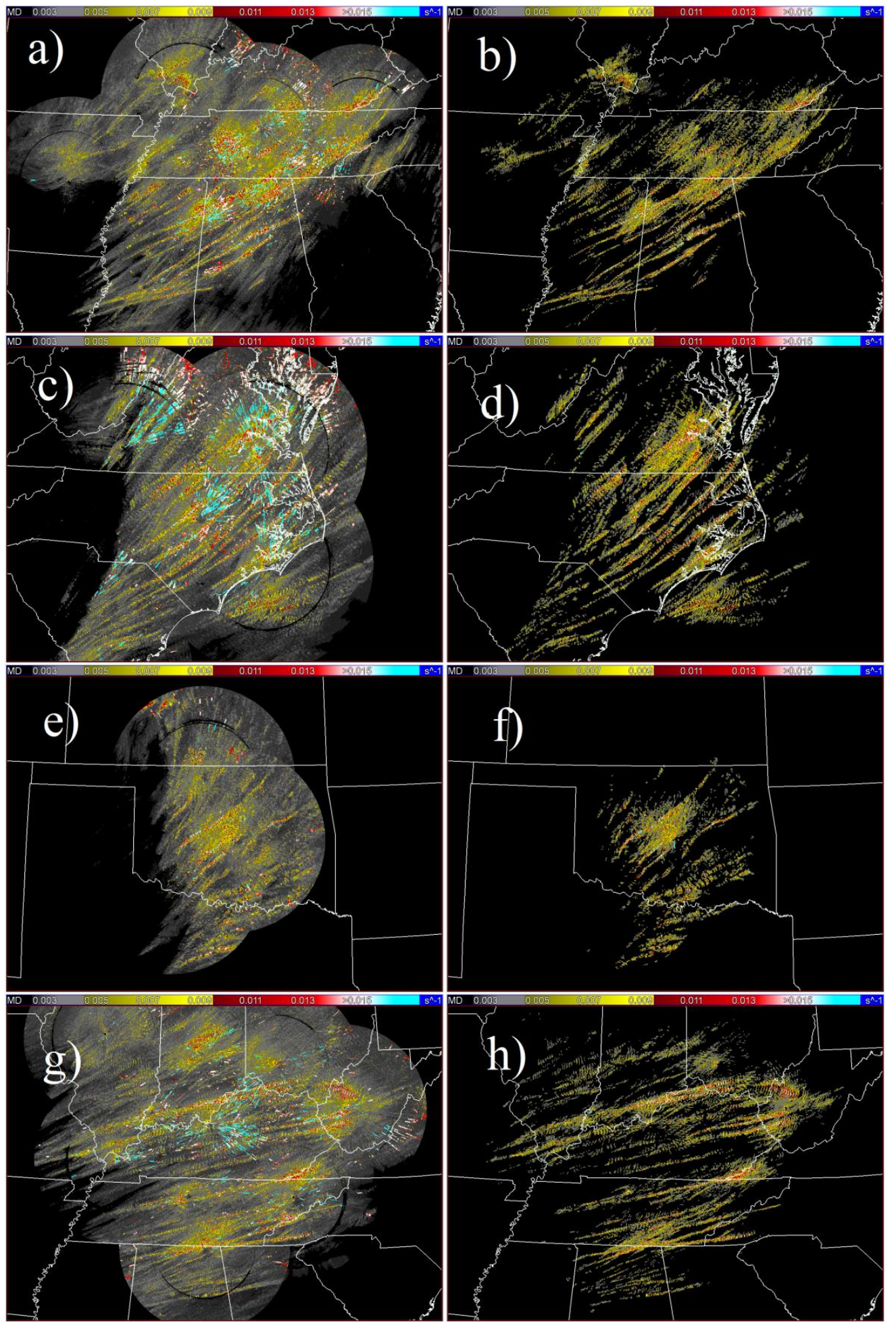

FIG. 16. The impacts of the quality control efforts on low level (0-3 km AGL) rotation

851 track products associated with four recent tornado events: the 27 April 2011 event before

852 (a) and after (b) quality control, the 16 April 2011 event before (c) and after (d) quality

853 control, the 24 May 2011 event before (e) and after (f) quality control, and the 2 March

8542012 event before ( $\mathrm{g}$ ) and after (h) quality control. 

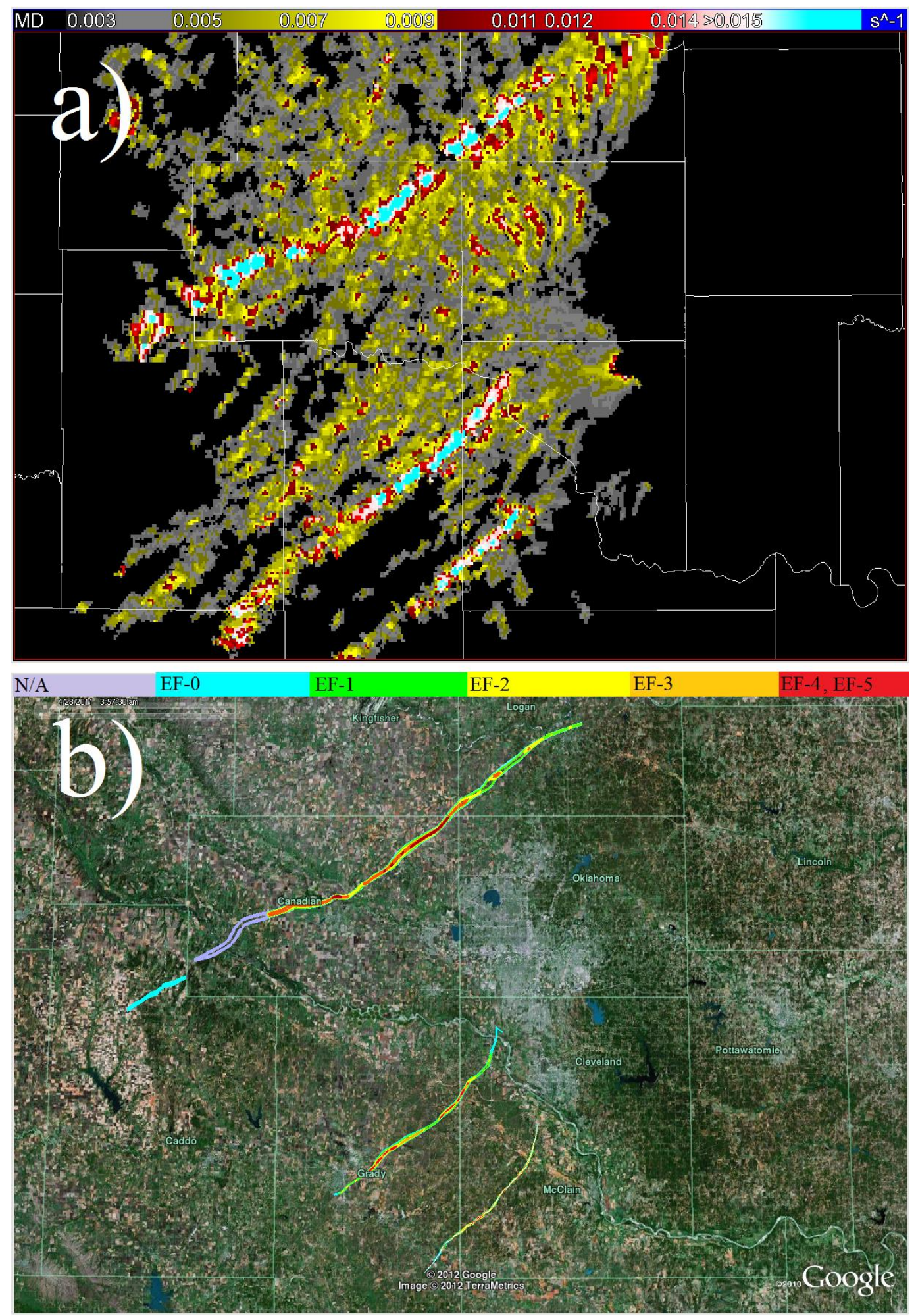

855

856

857

858

859

FIG. 17. (a) Low level (0-3 km AGL) rotation tracks over a 145-minute period associated with the 24 May 2011 tornado outbreak across central Oklahoma. (b) Plotted tornado EFscale ratings associated with tornadoes that occurred during that same time period as (a). Survey data provided courtesy of Kiel Ortega, Brandon Smith, and Gabe Garfield. 